\title{
An Efficient Adaptive Fuzzy Hierarchical Sliding Mode Control Strategy for 6 Degrees of Freedom Overhead Crane
}

This paper was downloaded from TechRxiv (https://www.techrxiv.org).

LICENSE

CC BY 4.0

SUBMISSION DATE / POSTED DATE

$29-12-2021 / 05-01-2022$

\section{CITATION}

Nguyen, Linh (2022): An Efficient Adaptive Fuzzy Hierarchical Sliding Mode Control Strategy for 6 Degrees of Freedom Overhead Crane. TechRxiv. Preprint. https://doi.org/10.36227/techrxiv.17704388.v1

DOI

10.36227/techrxiv.17704388.v1 


\title{
An Efficient Adaptive Fuzzy Hierarchical Sliding Mode Control Strategy for 6 Degrees of Freedom Overhead Crane
}

\author{
Minh Van Pham - Dung Manh Do · Hoang Viet Tran · Duy Hoang • \\ Hung Van Pham · Hai Xuan Le · Dong Quoc Hoang · Linh Nguyen
}

Received: date / Accepted: date

\begin{abstract}
The paper proposes a new approach to efficiently control a three-dimensional overhead crane with six degrees of freedom (DoF). In addition to five usual output variables including three positions of the trolley, bridge and pulley and two swing angles of the hoisting cable, it is proposed to consider elasticity of the hoisting cable, which causes oscillation in the cable direction. That is, there exists $6^{\text {th }}$ under-actuated output in the crane system. To design an efficient controller for the six-DoF crane, it first employs the hierarchical sliding mode control approach, which not only guarantees stability but also minimizes sway and oscillation of the overhead crane when it transports a payload to desired location. Moreover, the unknown and uncertain parameters of the system caused by its actuator nonlinearity and external disturbances are adaptively estimated and inferred by utilizing the fuzzy inference rule mechanism, which results in efficient operations of the crane in real time. More importantly, stabilization of the crane controlled by the proposed algorithm is
\end{abstract}

Minh Van Pham and Hung Van Pham

Faculty of Electrical Engineering, Hanoi University of Industry, Hanoi 10000, Vietnam

Dung Manh Do, Hoang Viet Tran and Duy Hoang

School of Electrical Engineering, Hanoi University of Science and Technology, Hanoi 10000, Vietnam

Hai Xuan Le

Institute of Technology, Hanoi University of Industry, Hanoi 10000, Vietnam

Dong Quoc Hoang

Vietnam Maritime University, Hai Phong 18000, Vietnam

Linh Nguyen

School of Engineering, IT and Physical Sciences, Federation University Australia, VIC 3350, Australia theoretically proved by the use of the Lyapunov function. The proposed control approach was implemented in the synthetic environment for the extensive evaluation, where the obtained results demonstrate its effectiveness.

Keywords 3D overhead crane - sliding mode control · fuzzy learning $\cdot 6$ degrees of freedom.

\section{Introduction}

Overhead crane systems have been playing a crucial role in many applications from industries or factories to transportation [1-5]. One of typical characteristics of an overhead crane is under-actuated. That is, in a crane system, the number of inputs is always smaller than that of outputs $[6,7]$. Having more outputs than inputs, an overhead system is constrained by strong nonlinearities, internal and external uncertainties and coupling properties. Hence, efficiently controlling an underactuated overhead crane with fewer independent control signals than its degrees of freedom is a fundamental but still challenging problem.

Due to its fundamentality, researchers, engineers and practitioners who design a control law for a underactuated crane system always concern about robustness in the system response due to its parameter uncertainties and actuator nonlinearities. To address the concern, sliding mode control (SMC) method has then been favoured for those systems [8-16]. For instance, the authors in [17-19] proposed the robust SMC controllers for a gantry crane, which allow the system with uncertain parameters and nonlinear actuators to robustly work under external disturbances in a working environment. It is noted that challenge in designing a robust SMC scheme for a under-actuated crane system is 
how to formulate sliding surfaces. In the work [20-22], the authors proposed to compute an intermediate variable from the state errors before a second-level sliding surface is formulated. Recently, the hierarchical SMC (HSMC) approach has been frequently utilized in defining the sliding surface for the SMC scheme in applications using under-actuated robotic systems [18, 19,23]. For instance, Wang et al. in their works [23] defined a first-level sliding surface for each subsystem of a a second-order under-actuated system. They then established the second-level sliding surface by simply adding the first-level sliding surfaces together.

Nonetheless, when developing a HSMC strategy for an overhead crane system, we have learned that if some parameters in the HSMC scheme are not properly chosen, it can severely causes vibration in the control system. Choosing an improper parameter for the HSMC controller is probably due to imprecision and imperfect information in the crane. This is very likely in practice since the under-actuated crane systems are constrained by their highly complicated nonlinearities and uncertainties. So far, it is well-known that fuzzy logic has been frequently exploited in addressing issues of inferring parameters for highly uncertain control systems [24]. For instance, the fuzzy logic has been extensively employed in controlling under-actuated systems [13,25]. By exploiting the Takagi-Sugeno fuzzy model, the authors in [26] to develop a closed-loop controller to automatically control operations of a crane with 2 degrees of freedom (DoF).

Regarding configuration of the cranes, to the best of our knowledge, most of the existing works consider a gantry system up to 5 DoF. For instance, in our previous work [1], a 2-dimensional (2D) crane with two output variables including the trolley position and swing angle is considered. A 3-dimensional (3D) overhead crane can have 5 DoF or output variables comprising positions of the trolley, bridge and pulley and swing angles of the hoisting cable in $x$ and $y$ directions. And in most of the considered crane systems, it is assumed that the hoisting cable is rigid. However, in practice the hoisting cable can be elastic. That is, there exists oscillation of the hoisting cable in the cable direction, which constitutes $6^{\text {th }}$ output variable or degree of freedom of the $3 \mathrm{D}$ crane. And this $6^{\text {th }}$ output component is also required to be controlled though it is under-actuated. It can now be seen that there are up to $6 \mathrm{DoF}$ in one $3 \mathrm{D}$ overhead crane, where there are 3 under-actuated output variables including two swing angles and oscillation of the hoisting cable in $x, y$, and the cable directions, respectively. A $3 \mathrm{D}$ overhead crane with $6 \mathrm{DoF}$ is the object to be controlled in this study.

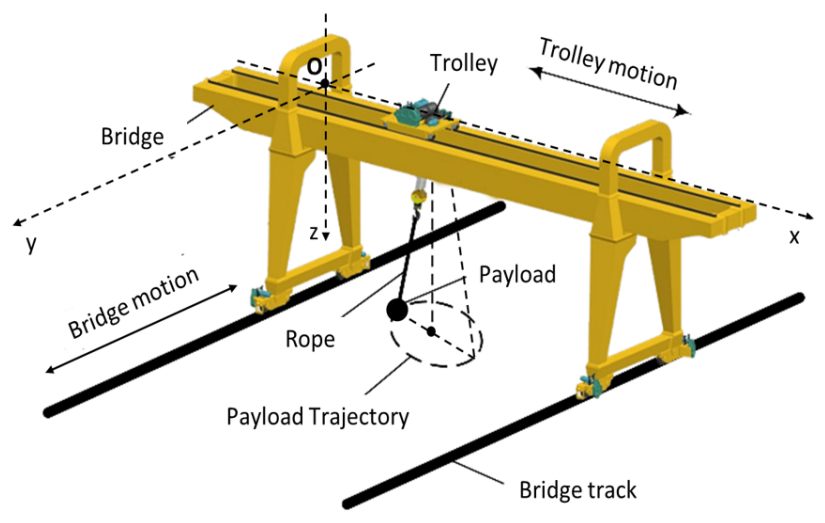

Fig. 1: A 3D overhead crane model [27].

Therefore, we take advantages of the HSMC approach, which guarantees robustness in the control performance, to design a controller for a 3D overhead crane with 6 DoF. In the control design, it is assumed that the parameters in the HSMC law are deterministic. Nonetheless, in fact due to the nonlinear and uncertain properties, determining those parameters in the 6 DoF crane system is impractical. Moreover, if those parameters are not properly chosen, it can lead to severe vibration in the control system. To this end, we propose to exploit the fuzzy inference rule system to adaptively infer the parameters of the HSMC scheme over time. It is noted that the fuzzy logic can effectively estimate the parameters of a system given its imprecision and imperfect information. More importantly, stability of the control performance in the 3D under-actuated crane obtained by the proposed approach is theoretically proved by the Lyapunov theory. We then extensively evaluated our algorithm in three typical scenarios in operating an overhead crane, where the obtained results are highly promising.

The remaining of the paper is arranged as follows. A model of a 3D overhead crane system with 6 DoF is introduced in Section 2. Section 3 presents how to design a HSMC law for the 6 DoF crane systems while Section 4 discusses how to adaptively learn its parameters by the use of the fuzzy logic. The results of evaluating the proposed approach are compared and summarised in Section 5 before conclusions are drawn in Section 6 . It is noted that in this work matrices and vectors are expressed in square brackets [.] or parentheses $(\cdot)$ while long expressions are encapsulated in braces $\{\cdot\}$. 


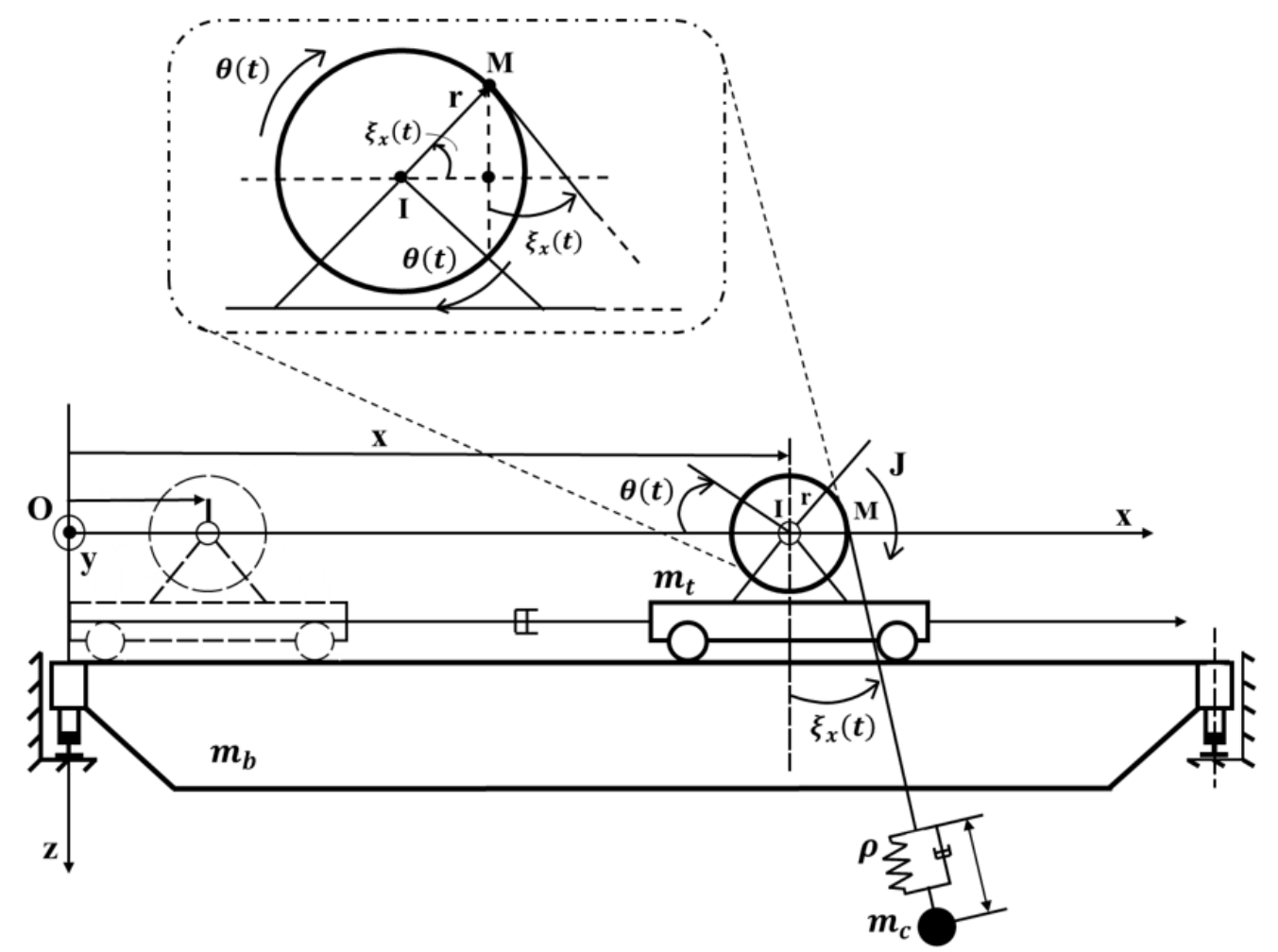

Fig. 2: A digram of a 3D overhead crane model presented in Oxz plane.

\section{A Model of 3D Overhead Crane with Six Degrees of Freedom}

Let us consider a three-dimensional (3D) overhead crane as demonstrated in Fig. 1. It is assumed that the trolley moves along $x$ direction while the bridge moves along $y$ direction. A pulley embedded on the trolley, as can be seen in Fig. 2, plays a role of pulling a payload up and down along $z$ direction through a hoisting cable. Operationally, the trolley, bridge and pulley cooperatively allows the crane to transport an object from one location to another.

Let $x(t)$ and $y(t)$ denote displacements of the trolley and bridge, respectively. We define $\theta(t)$ as a rotation angle of the pulley. It is noted that under motions of the trolley, bridge and pulley, a payload hooked to the hoisting cable swings around its equilibrium point. If we define $O$ as origin of a Cartesian coordinate, then sway of the payload in the planes $O x z$ and $O y z$ is denoted by $\xi_{x}(t)$ and $\xi_{y}(t)$, respectively. More importantly, in this work, we consider elasticity of the hoisting cable. In fact, in many crane systems, the hoisting cable is not rigid but elastic. Therefore, we define $\gamma(t)$ as oscillation of the hoisting cable in the cable direction due to the elasticity. It can be seen the overhead crane system has six variables including $x(t), y(t), \theta(t), \xi_{x}(t), \xi_{y}(t)$ and $\gamma(t)$, presenting its six degrees of freedom (DoF). While $x(t), y(t)$ and $\theta(t)$ can be directly controlled by the trolley, bridge and pulley, there is no actuator having direct connection with $\xi_{x}(t), \xi_{y}(t)$ and $\gamma(t)$. That is, $\xi_{x}(t), \xi_{y}(t)$ and $\gamma(t)$ are considered as under-actuated states of the crane.

We denote $x_{d}(t), y_{d}(t)$ and $\theta_{d}(t)$ as desired references of the actuated states $x(t), y(t)$ and $\theta(t)$, respectively. And the under-actuated states $\xi_{x}(t), \xi_{y}(t)$ and $\gamma(t)$ are expected to reach zero during operations of the crane. Thus,

$$
\begin{aligned}
& \lim _{t \rightarrow+\infty}|\sup (x(t))|=x_{d}(t) \\
& \lim _{t \rightarrow+\infty}\left|\sup \left(\xi_{x}(t)\right)\right|=0 \\
& \lim _{t \rightarrow+\infty}|\sup (y(t))|=y_{d}(t) \\
& \lim _{t \rightarrow+\infty}\left|\sup \left(\xi_{y}(t)\right)\right|=0 \\
& \lim _{t \rightarrow+\infty}|\sup (\theta(t))|=\theta_{d}(t) \\
& \lim _{t \rightarrow+\infty}|\sup (\gamma(t))|=0
\end{aligned}
$$

For the simplicity purpose, we let $\underline{\chi}(t)=\left[\begin{array}{llllll}x(t) & y(t) & \theta(t) & \xi_{x}(t) & \xi_{y}(t) & \gamma(t)\end{array}\right]^{T}$ denote a state vector of the $3 \mathrm{D}$ crane.

Apart from the hoisting cable, it is assumed that other components in the crane is rigid, which only depend on the holonomic internal kinematic constraints. 
Ratio of the pulley is set to 1 while gravity $g=9,81 \mathrm{~m} / \mathrm{s}^{2}$ Masses of the bridge, payload and trolley-pulley combination are defined by $m_{b}, m_{c}$ and $m_{t}$, respectively. While the elastic coefficient of the hoisting cable is defined by $\rho$, we denote $J$ and $r$ as inertia and radius of the pulley. Hence, the potential energy of the overhead crane is computed by

$\mathrm{E}(t)=\left\{\begin{array}{l}-m_{c} g r c o s\left(\xi_{y}(t)\right) \sin \left(\xi_{x}(t)\right)+\frac{1}{2} \rho \gamma^{2}+ \\ +m_{c} g(\gamma(t)+\Delta \gamma) \cos \left(\xi_{x}(t)\right) \times \\ \times \cos \left(\xi_{y}(t)\right)+m_{c} g r\left(\theta(t)+\xi_{x}(t)\right) \times \\ \times \cos \left(\xi_{x}(t)\right) \cos \left(\xi_{y}(t)\right)\end{array}\right\}$.

And the energy loss caused by frictions is given as follows.

$\Phi(t)=\frac{1}{2} \underline{\varpi}(t)^{T} D \underline{\varpi}(t)$,

where $\underline{\varpi}(t)=(\dot{\theta}(t) \dot{\gamma}(t) \dot{x}(t) \dot{y}(t))^{T}$ and

$D=\left(\begin{array}{cccc}\eta_{m} & 0 & 0 & 0 \\ 0 & \eta_{r} & 0 & 0 \\ 0 & 0 & \eta_{t} & 0 \\ 0 & 0 & 0 & \eta_{b}\end{array}\right)$ is a matrix of the damping coefficients.

Moreover, the kinetic energy $T(t)$ of the crane is a sum of the kinetic energy of the pulley $T_{r}(t)$, the kinetic energy of the bridge $T_{b}(t)$, the kinetic energy of the trolley $T_{t}(t)$ and the kinetic energy of the load $T_{c}(t)$.

$T(t)=T_{c}(t)+T_{t}(t)+T_{b}(t)+T_{r}(t)$,

where

$T_{r}(t)=\frac{1}{2} J\left(\frac{d(\theta(t))}{d t}\right)^{2}$,

$T_{b}(t)=\frac{1}{2} m_{b}\left(\frac{d(y(t))}{d t}\right)^{2}$,

$T_{t}(t)=\frac{1}{2} m_{t}\left[\left(\frac{d}{d t} x(t)\right)^{2}+\left(\frac{d}{d t} y(t)\right)^{2}\right]$,

$T_{c}(t)=\frac{1}{2} m_{c}\left(V_{c x}^{2}+V_{c y}^{2}+V_{c z}^{2}\right)$.

It is noted that $V_{c x}, V_{c y}$ and $V_{c z}$ are velocities of the payload in $x, y$ and $z$ directions, respectively, where they can be calculated as follows.

$V_{c x}(t)=\left\{\begin{array}{l}\dot{x}(t)+\dot{\gamma}(t) \sin \left(\xi_{x}(t)\right)+ \\ +r\left(\dot{\theta}(t)+\dot{\xi}_{x}(t)\right) \sin \left(\xi_{x}(t)\right)+ \\ +(\gamma(t)+\Delta \gamma) \dot{\xi}_{x}(t) \cos \left(\xi_{x}(t)\right)+ \\ +\left(r \xi_{x}(t)\right) \dot{\xi}_{x}(t) \cos \left(\xi_{x}(t)\right)+ \\ +r \theta(t) \dot{\xi}_{x}(t) \cos \left(\xi_{x}(t)\right)- \\ -r \dot{\xi}_{x}(t) \sin \left(\xi_{x}(t)\right)\end{array}\right\}$.

$$
\begin{aligned}
& V_{c y}(t)= \\
& =\left\{\begin{array}{l}
\dot{y}(t)+\dot{\gamma}(t) \sin \left(\xi_{y}(t)\right) \cos \left(\xi_{x}(t)\right)+ \\
+r\left(\dot{\theta}(t)+\dot{\xi}_{x}(t)\right) \sin \left(\xi_{y}(t)\right) \cos \left(\xi_{x}(t)\right)+ \\
+(\gamma(t)+\Delta \gamma) \dot{\xi}_{y}(t) \cos \left(\xi_{x}(t)\right) \cos \left(\xi_{y}(t)\right)- \\
-(\gamma(t)+\Delta \gamma) \dot{\xi}_{x}(t) \sin \left(\xi_{x}(t)\right) \sin \left(\xi_{y}(t)\right)+ \\
+r \xi_{x}(t) \dot{\xi}_{y}(t) \cos \left(\xi_{x}(t)\right) \cos \left(\xi_{y}(t)\right)+ \\
+r \theta(t) \cos \left(\xi_{x}(t)\right) \cos \left(\xi_{y}(t)\right)- \\
-r \xi_{x}(t) \dot{\xi}_{x}(t) \sin \left(\xi_{x}(t)\right) \sin \left(\xi_{y}(t)\right)- \\
-r \theta(t) \dot{\xi}_{x}(t) \sin \left(\xi_{x}(t)\right) \sin \left(\xi_{y}(t)\right)
\end{array}\right\} .
\end{aligned}
$$

$$
\begin{aligned}
& V_{c z}(t)= \\
& =\left\{\begin{array}{l}
\dot{\gamma}(t) \cos \left(\xi_{y}(t)\right) \cos \left(\xi_{x}(t)\right)+ \\
+r\left(\dot{\theta}(t)+\dot{\xi}_{x}(t)\right) \cos \left(\xi_{y}(t)\right) \cos \left(\xi_{x}(t)\right)- \\
-(\gamma(t)+\Delta \gamma) \dot{\xi}_{y}(t) \cos \left(\xi_{x}(t)\right) \sin \left(\xi_{y}(t)\right)- \\
-(\gamma(t)+\Delta \gamma) \dot{\xi}_{x}(t) \sin \left(\xi_{x}(t)\right) \cos \left(\xi_{y}(t)\right)- \\
-r \xi_{x}(t) \dot{\xi}_{y}(t) \cos \left(\xi_{x}(t)\right) \sin \left(\xi_{y}(t)\right)- \\
-r \theta(t) \dot{\xi}_{y}(t) \cos \left(\xi_{x}(t)\right) \sin \left(\xi_{y}(t)\right)- \\
-r \xi_{x}(t) \dot{\xi}_{x}(t) \sin \left(\xi_{x}(t)\right) \cos \left(\xi_{y}(t)\right)- \\
-r \theta(t) \dot{\xi}_{x}(t) \sin \left(\xi_{x}(t)\right) \cos \left(\xi_{y}(t)\right)- \\
-r \dot{\xi}_{x}(t) \cos \left(\xi_{y}(t)\right) \cos \left(\xi_{x}(t)\right)+ \\
+r \dot{\xi}_{y}(t) \sin \left(\xi_{x}(t)\right) \sin \left(\xi_{y}(t)\right)
\end{array}\right\} .
\end{aligned}
$$

By exploiting the Euler-Lagrange equation, the crane system can be presented in the differential form as follows.

$$
\begin{aligned}
\frac{d}{d t}\left[\left(\frac{\partial L(\underline{\chi}, t)}{\partial \underline{\dot{\chi}}(t)}\right)\right]^{T} & -\left[\frac{\partial L(\underline{\chi}, t)}{\partial \underline{\chi}(t)}\right]^{T} \\
& =\underline{u}(t)-\left[\frac{\partial \Phi(\underline{\chi}, t)}{\partial \underline{\dot{\chi}}(t)}\right]^{T},
\end{aligned}
$$

where

$L=L(\underline{\chi}, t)=T(\underline{\chi}, t)-E(\underline{\chi}, t)$

is the Lagrange function.

$\underline{\dot{\chi}}(t)=\left(\dot{x}(t) \dot{y}(t) \dot{\theta}(t) \dot{\xi}_{x}(t) \dot{\xi}_{y}(t) \dot{\gamma}(t)\right)^{T}$ is a vector of the first order derivative of the system states. $\underline{u}(t)=$ $\left(u_{1}(t) u_{2}(t) u_{3}(t) 0000 \begin{array}{llll} & 0 & 0\end{array}\right)^{T}$ is a vector of the input control signals. It is noticed that $u_{1}(t), u_{2}(t)$ and $u_{3}(t)$ are the input signals to control the trolley, bridge and pulley, respectively. The the Jacobian derivatives of the 
Lagrange function can be computed by

$$
\frac{\partial L(\underline{\chi}, t)}{\partial \underline{\chi}(t)}=\left[\begin{array}{c}
\frac{\partial L}{\partial x(t)} \\
\frac{\partial L}{\partial y(t)} \\
\frac{\partial L}{\partial \theta(t)} \\
\frac{\partial L}{\partial \xi_{x}(t)} \\
\frac{\partial L}{\partial \xi_{y}(t)} \\
\frac{\partial L}{\partial \gamma(t)}
\end{array}\right]^{T}, \quad \frac{\partial L(\underline{\chi}, t)}{\partial \underline{\dot{\chi}}(t)}=\left[\begin{array}{c}
\frac{\partial L}{\partial \dot{x}(t)} \\
\frac{\partial L}{\partial \dot{y}(t)} \\
\frac{\partial L}{\partial \dot{\theta}(t)} \\
\frac{\partial L}{\partial \dot{\xi}_{x}(t)} \\
\frac{\partial L}{\partial \dot{\xi}_{y}(t)} \\
\frac{\partial L}{\partial \dot{\gamma}(t)}
\end{array}\right]^{T}
$$

Therefore, the dynamic model of the 3D overhead crane with 6 DoF can be represented by

$M(\underline{\chi}, t) \underline{\ddot{\chi}}+(C(\underline{\chi}, \underline{\dot{\chi}}, t)+D) \underline{\dot{\chi}}(t)+G(\underline{\chi}, t)=\underline{u}(t)$,

where $\underline{\underline{\chi}}(t)=\left(\ddot{x}(t) \ddot{y}(t) \ddot{\theta}(t) \ddot{\xi}_{x}(t) \ddot{\xi}_{y}(t) \ddot{\gamma}(t)\right)^{T}$ is a vector of the second order derivatives of the system states. $M(\underline{\chi}, t)=\left(\begin{array}{llllll}m_{11} & m_{12} & m_{13} & m_{14} & m_{15} & m_{16} \\ m_{21} & m_{22} & m_{23} & m_{24} & m_{25} & m_{26} \\ m_{31} & m_{32} & m_{33} & m_{34} & m_{35} & m_{36} \\ m_{41} & m_{42} & m_{43} & m_{44} & m_{45} & m_{46} \\ m_{51} & m_{52} & m_{53} & m_{54} & m_{55} & m_{56} \\ m_{61} & m_{62} & m_{63} & m_{64} & m_{65} & m_{66}\end{array}\right)>0$.

$C(\underline{\chi}, \underline{\dot{\chi}})=\left(\begin{array}{llllll}c_{11} & c_{12} & c_{13} & c_{14} & c_{15} & c_{16} \\ c_{21} & c_{22} & c_{23} & c_{24} & c_{25} & c_{26} \\ c_{31} & c_{32} & c_{33} & c_{34} & c_{35} & c_{36} \\ c_{41} & c_{42} & c_{43} & c_{44} & c_{45} & c_{46} \\ c_{51} & c_{52} & c_{53} & c_{54} & c_{55} & c_{56} \\ c_{61} & c_{62} & c_{63} & c_{64} & c_{65} & c_{66}\end{array}\right)$ is the Coriolis co-

efficient matrix while $G(\underline{\chi}, t)=\left(\begin{array}{llllll}0 & 0 & g_{1} & g_{2} & g_{3} & g_{4}\end{array}\right)^{T}$ is the gravitational matrix. Calculation details of elements of $M(\underline{\chi}, t), C(\underline{\chi}, \underline{\dot{\chi}})$ and $G(\underline{\chi}, t)$ can be found in Appendix.

\section{Hierarchical Sliding Mode Controller for 3D Overhead Crane}

In this section, we will present how to design a hierarchical sliding mode control (HSMC) scheme for a $3 \mathrm{D}$ overhead crane with 6 DoF. It is noted that we exploit the dynamic model of the crane as introduced in Section 2 .

$$
\text { Let us split } \underline{\chi}(t) \text { into } \underline{\chi}(t)=\left(\begin{array}{ll}
\underline{\chi}_{1}(t) \quad \underline{\chi}_{2}(t) & )^{T},
\end{array}\right.
$$
where $\underline{\chi}_{1}(t)=\left(\begin{array}{c}x(t) \\ y(t) \\ \theta(t)\end{array}\right), \underline{\dot{\chi}}_{1}(t)=\left(\begin{array}{c}\dot{x}(t) \\ \dot{y}(t) \\ \dot{\theta}(t)\end{array}\right)$ and $\underline{\chi}_{1}(t)=\left(\begin{array}{c}\ddot{x}(t) \\ \ddot{y}(t) \\ \ddot{\theta}(t)\end{array}\right)$ are the corresponding vectors of the actuated states while $\underline{\chi}_{2}(t)=\left(\begin{array}{c}\xi_{x}(t) \\ \xi_{y}(t) \\ \gamma(t)\end{array}\right)$,

$\underline{\chi}_{2}(t)=\left(\begin{array}{c}\dot{\xi}_{x}(t) \\ \dot{\xi}_{y}(t) \\ \dot{\gamma}(t)\end{array}\right)$ and $\ddot{\chi}_{2}(t)=\left(\begin{array}{c}\ddot{\xi}_{x}(t) \\ \ddot{\xi}_{y}(t) \\ \ddot{\gamma}(t)\end{array}\right)$ are the corresponding vectors of the under-actuated states, respectively.

$\underline{u}(t)=\left(\begin{array}{ll}\underline{\tau}(t) & \underline{0}\end{array}\right)^{T}$ in which $\underline{\tau}(t)=\left(\begin{array}{l}u_{1}(t) \\ u_{2}(t) \\ u_{3}(t)\end{array}\right) \in \mathbb{R}^{3 \times 1}$

We now define

$K(\underline{\chi}, t)=M^{-1}(\underline{\chi}, t)=\left(\begin{array}{l}K_{1}(\underline{\chi}, t) K_{3}(\underline{\chi}, t) \\ K_{2}(\underline{\underline{\chi}}, t) K_{4}(\underline{\underline{\chi}}, t)\end{array}\right)$ with $K_{1}(\underline{\chi}, t), K_{2}(\underline{\chi}, t), K_{3}(\underline{\chi}, t)$ and $K_{4}(\underline{\chi}, t) \in \mathbb{R}^{3 \times 3}$.

And $C(\underline{\chi}, \underline{\dot{\chi}}, t)=\left(\begin{array}{l}C_{1}(\underline{\chi}, \underline{\dot{\chi}}, t) C_{3}(\underline{\chi}, \underline{\dot{\chi}}, t) \\ C_{2}(\underline{\bar{\chi}}, \underline{\dot{\chi}}, t) C_{4}(\underline{\underline{\chi}}, \underline{\dot{\chi}}, t)\end{array}\right)$ with

$C_{1}(\underline{\chi}, \underline{\dot{\chi}}, t), C_{2}(\underline{\chi}, \underline{\dot{\chi}}, t), C_{3}(\underline{\chi}, \underline{\dot{\chi}}, t)$, and

$C_{4}(\underline{\chi}, \underline{\dot{\chi}}, t) \in \mathbb{R}^{3} \overline{\times}$.

The block matrices can be computed by

$$
\begin{aligned}
& C_{1}(\underline{\chi}, \underline{\dot{\chi}}, t)=\left(\begin{array}{lll}
c_{11} & c_{12} & c_{13} \\
c_{21} & c_{22} & c_{23} \\
c_{31} & c_{32} & c_{33}
\end{array}\right), \\
& C_{2}(\underline{\chi}, \underline{\dot{\chi}}, t)=\left(\begin{array}{lll}
c_{41} & c_{42} & c_{43} \\
c_{51} & c_{52} & c_{53} \\
c_{61} & c_{62} & c_{63}
\end{array}\right),
\end{aligned}
$$

$C_{3}(\underline{\chi}, \underline{\dot{\chi}}, t)=\left(\begin{array}{lll}c_{14} & c_{15} & c_{16} \\ c_{24} & c_{25} & c_{26} \\ c_{34} & c_{35} & c_{36}\end{array}\right)$

$C_{4}(\underline{\chi}, \underline{\dot{\chi}}, t)=\left(\begin{array}{lll}c_{44} & c_{45} & c_{46} \\ c_{54} & c_{55} & c_{56} \\ c_{64} & c_{65} & c_{66}\end{array}\right)$.

Moreover,

$G(\underline{\chi}, t)=\left(\begin{array}{l}G_{1}(\underline{\chi}, t) \\ G_{2}(\underline{\chi}, t)\end{array}\right)$,

where

$G_{1}(\underline{\chi}, t)=\left(\begin{array}{c}0 \\ 0 \\ g_{1}\end{array}\right), \quad G_{2}(\underline{\chi}, t)=\left(\begin{array}{l}g_{2} \\ g_{3} \\ g_{4}\end{array}\right)$.

Therefore,

$\underline{\ddot{\chi}}_{1}(t)=\underline{f_{1}}(\underline{\chi}, \underline{\dot{\chi}}, t)+K_{1}(\underline{\chi}, t) \underline{\tau}$ 
$\underline{\ddot{\chi}}_{2}(t)=\underline{f_{2}}(\underline{\chi}, \underline{\dot{\chi}}, t)+K_{2}(\underline{\chi}, t) \underline{\tau}$

where

$\underline{f_{1}}(\underline{\chi}, \underline{\chi}, t)=-\left\{\begin{array}{l}l\left(K_{1}(\underline{\chi}, t) G_{1}(\underline{\chi}, t)+\right. \\ \left.+K_{3}(\underline{\chi}, t) G_{2}(\underline{\chi}, t)\right)+ \\ +K_{1}(\underline{\chi}, t) C_{1}(\underline{\chi}, \underline{\dot{\chi}}, t) \underline{\dot{\chi}}_{1}(t)+ \\ +K_{3}(\underline{\chi}, t) C_{2}(\underline{\chi}, \underline{\dot{\chi}}, t) \underline{\dot{\chi}}_{1}(t)+ \\ +K_{1}(\underline{\chi}, t) C_{3}(\underline{\chi}, \underline{\dot{\chi}}, t) \underline{\dot{\chi}}_{2}(t)+ \\ +K_{3}(\underline{\chi}, t) C_{4}(\underline{\chi}, \underline{\dot{\chi}}, t) \underline{\dot{\chi}}_{2}(t)\end{array}\right\}$,

$\underline{f_{2}}(\underline{\chi}, \underline{\chi}, t)=-\left\{\begin{array}{l}l\left(K_{2}(\underline{\chi}, t) G_{1}(\underline{\chi}, t)+\right. \\ \left.+K_{4}(\underline{\chi}, t) G_{2}(\underline{\chi}, t)\right)+ \\ +K_{2}(\underline{\chi}, t) C_{1}(\underline{\chi}, \underline{\dot{\chi}}, t) \dot{\dot{\chi}}_{1}(t)+ \\ +K_{4}(\underline{\chi}, t) C_{2}(\underline{\chi}, \underline{\dot{\chi}}, t) \underline{\dot{\chi}}_{1}(t)+ \\ +K_{2}(\underline{\chi}, t) C_{3}(\underline{\chi}, \underline{\dot{\chi}}, t) \underline{\dot{\chi}}_{2}(t)+ \\ +K_{4}(\underline{\chi}, t) C_{4}(\underline{\chi}, \underline{\dot{\chi}}, t) \underline{\dot{\chi}}_{2}(t)\end{array}\right\}$

The model in (16) and (17) can be represented as a state model by

$\underline{\dot{x}}_{1}(t)=\underline{x}_{2}(t)$,

$\underline{\dot{x}}_{2}(t)=\underline{f_{1}}(\underline{\chi}, \underline{\dot{\chi}}, t)+K_{1}(\underline{\chi}, t) \underline{\tau}$,

$\underline{\dot{x}}_{3}(t)=\underline{x}_{4}(t)$,

$\underline{\dot{x}}_{4}(t)=\underline{f_{2}}(\underline{\chi}, \underline{\dot{\chi}}, t)+K_{2}(\underline{\chi}, t) \underline{\tau}$.

Let

$\underline{e}_{1}(t)=\underline{x}_{1}(t)-\underline{x}_{1 d}(t)$

$\underline{e}_{3}(t)=\underline{x}_{3}(t)-\underline{x}_{3 d}(t)$

denote errors between the output signals $\left(\underline{x}_{1}(t), \underline{x}_{3}(t)\right)$ and the desired references $\left(\underline{x}_{1 d}(t), \underline{x}_{3 d}(t)\right) . \underline{x}_{1 d}(t)=$ $\left(\begin{array}{l}x_{d} \\ y_{d} \\ \theta_{d}\end{array}\right)$ is a vector of the desired references of the actuated states while $\underline{x}_{3 d}(t)=\left(\begin{array}{l}0 \\ 0 \\ 0\end{array}\right)$ is a vector of the desired references of the under-actuated states. The errors can now be specified by

$\underline{\dot{e}}_{1}(t)=\underline{e}_{2}(t)$,

$\underline{\dot{e}}_{2}(t)=\underline{f_{1}}(\underline{\chi}, \underline{\dot{\chi}}, t)+K_{1}(\underline{\chi}, t) \underline{\tau}-\underline{\ddot{x}}_{1 d}(t)$,

$\underline{\dot{e}}_{3}(t)=\underline{e}_{4}(t)$,

$\underline{\dot{e}}_{4}(t)=\underline{f_{2}}(\underline{\chi}, \underline{\dot{\chi}}, t)+K_{2}(\underline{\chi}, t) \cdot \underline{\tau}$
In order to minimize the errors in (24) - (27), the control strategy is proposed as follows.

$\underline{\tau}(t)=\left\{\begin{array}{l}\underline{\tau}_{1 e q}(t)+ \\ +\underline{\tau}_{2 e q}(t)-\left(\lambda_{1} K_{1}(\underline{\chi}, t)+\lambda_{2} K_{2}(\underline{\chi}, t)\right)^{-1} \times \\ \times\left\{\begin{array}{l}\lambda_{1} K_{1}(\underline{\chi}, t) \underline{\tau}_{2 e q}(t)+ \\ +\lambda_{2} K_{2}(\underline{\chi}, t) \underline{\tau}_{1 e q}(t)+ \\ +\eta_{3} \underline{s}(t)+\eta_{4} \operatorname{sign}(\underline{s}(t))\end{array}\right\}\end{array}\right\}$

where $\lambda_{1}, \lambda_{2}, \alpha_{1}, \alpha_{2} \in \mathbb{R}^{3 \times 3}$ are the symmetric, positivedefinite and constant matrices while $\eta_{3}$ and $\eta_{4}$ are the symmetric and positive-definite matrices. The secondlevel sliding surface is compiled by two first-level sliding surfaces from two subsystems as follows.

$\underline{s}(t)=\lambda_{1} \underline{s}_{1}(t)+\lambda_{2} \underline{s}_{2}(t)$,

where

$\underline{s}_{1}(t)=\alpha_{1} \underline{e}_{1}(t)+\underline{e}_{2}(t)$.

$\underline{s}_{2}(t)=\alpha_{2} \underline{e}_{3}(t)+\underline{e}_{4}(t)$.

Thus, two control signals for two subsystems are given by

$\underline{\tau}_{1 e q}=-K_{1}^{-1}(\underline{\chi}, t)\left(\alpha_{1} \underline{e}_{2}(t)+\underline{f_{1}}(\underline{\chi}, \underline{\dot{\chi}}, t)-\underline{\ddot{x}}_{1 d}(t)\right)$,

$\underline{\tau}_{2 e q}=-K_{2}^{-1}(\underline{\chi}, t)\left(\alpha_{2} \underline{e}_{4}(t)+\underline{f_{2}}(\underline{\chi}, \underline{\dot{\chi}}, t)\right)$.

Stability of the proposed control law can be specified by the following lemma.

Lemma 1 The proposed control scheme (28) guarantees stability of the $3 D$ overhead crane with 6 DoF, which leads to $\lim _{t \rightarrow+\infty} \underline{e}_{j}(t)=\underline{0}, j=1 \div 4$.

Proof Let us consider the first subsystem represented by

$\underline{\dot{x}}_{1}(t)=\underline{x}_{2}(t)$,

$\underline{\dot{x}}_{2}(t)=\underline{f_{1}}(\underline{\chi}, \underline{\dot{\chi}}, t)+K_{1}(\underline{\chi}, t) \underline{\tau}_{1}$,

where its sliding surface is

$\underline{s}_{1}(t)=\alpha_{1} \underline{e}_{1}(t)+\underline{e}_{2}(t)$.

To obtain $\lim _{t \rightarrow+\infty} \underline{s}_{1}(t)=\underline{0}$, we consider the Lyapunov function candidate of $\underline{s}_{1}(t)$ as follows.

$\underline{V}_{1}\left(\underline{s}_{1}(t)\right)=\frac{1}{2} \underline{s}_{1}^{T}(t) \underline{s}_{1}(t)$. 
Taking derivative both side of equation (33), it yields

$\underline{\dot{V}}_{1}(t)=\underline{s}_{1}^{T}(t) \underline{\dot{s}}_{1}(t)$

$=\underline{s}_{1}^{T}(t)\left(\alpha_{1} \underline{e}_{2}(t)+\underline{f_{1}}(\underline{\chi}, \underline{\dot{\chi}}, t)+K_{1}(\underline{\chi}, t) \underline{\tau}_{1}-\underline{\ddot{x}}_{1 d}(t)\right)$

The control signal for the first subsystem includes the equivalent control law $\underline{\tau}_{1 e q}(t)$ and the switch control scheme $\underline{\tau}_{1 s w}(t)$. Hence,

$\underline{V}_{1}(t)=\underline{s}_{1}^{T}(t)\left\{\begin{array}{l}\alpha_{1} \underline{e}_{2}(t)+\underline{f_{1}}(\underline{\chi}, \underline{\dot{\chi}}, t)+ \\ +K_{1}(\underline{\chi}, t)\left(\underline{\tau}_{1 e q}+\underline{\tau}_{1 s w}\right)- \\ -\underline{\ddot{x}}_{1 d}(t)\end{array}\right\}$.

That is,

$\underline{V}_{1}(t)=\underline{s}_{1}^{T}(t) \times$

$\times\left\{\begin{array}{l}\alpha_{1} \underline{e}_{2}(t)+\underline{f}_{1}(\underline{\chi}, \underline{\dot{\chi}}, t)+K_{1}(\underline{\chi}, t) \underline{\tau}_{1 e q}-\underline{\ddot{x}}_{1 d}(t)+ \\ +K_{1}(\underline{\chi}, t) \underline{\tau}_{1 s \mathrm{w}}+\eta_{1} \underline{s}_{1}(t)+\eta_{2} \operatorname{sign}\left(\underline{s}_{1}(t)\right)- \\ -\eta_{1} \underline{s}_{1}(t)-\eta_{2} \operatorname{sign}\left(\underline{s}_{1}(t)\right)\end{array}\right\}$,

where $\eta_{1}$ and $\eta_{2}$ are the symmetric and positive-definite matrices. If

$$
\begin{aligned}
& \left\{\begin{array}{l}
K_{1}(\underline{\chi}, t) \underline{\tau}_{1 e q}+\alpha_{1} \underline{e}_{2}(t)+\underline{f_{1}}(\underline{\chi}, \underline{\dot{\chi}}, t)-\underline{\ddot{x}}_{1 d}(t)=\underline{0} \\
K_{1}(\underline{\chi}, t) \underline{\tau}_{1 s w}(t)+\eta_{1} \underline{s}_{1}(t)+\eta_{2} \operatorname{sign}\left(\underline{s}_{1}(t)\right)=\underline{0}
\end{array}\right. \\
& \Leftrightarrow\left\{\begin{aligned}
\underline{\tau}_{1 e q}= & -K_{1}^{-1}(\underline{\chi}, t) \times \\
& \times\left(\alpha_{1} \underline{e}_{2}(t)+\underline{f_{1}}(\underline{\chi}, \underline{\dot{\chi}}, t)-\underline{\ddot{x}}_{1 d}(t)\right), \\
\underline{\tau}_{1 s w}(t)= & -K_{1}{ }^{-1}(\underline{\chi}, t) \times \\
& \times\left(\eta_{1} \underline{s}_{1}(t)+\eta_{2} \operatorname{sign}\left(\underline{s}_{1}(t)\right)\right),
\end{aligned}\right.
\end{aligned}
$$

then one has

$\underline{\dot{V}}_{1}(t)=-\underline{s}_{1}^{T}(t) \eta_{1} \underline{s}_{1}(t)-\underline{s}_{1}^{T}(t) \eta_{2} \operatorname{sign}\left(\underline{s}_{1}(t)\right) \leq 0$,

which guarantees stability of the first subsystem.

Likewise, the second subsystem

$\underline{\dot{e}}_{3}(t)=\underline{e}_{4}(t)$,

$\underline{\dot{e}}_{4}(t)=\underline{f_{2}}(\underline{\chi}, \underline{\dot{\chi}}, t)+K_{2}(\underline{\chi}, t) \underline{\tau}_{2}$,

where its sliding surface is

$\underline{s}_{2}(t)=\alpha_{3} \underline{e}_{3}(t)+\underline{e}_{4}(t)$,

also holds

$K_{2}(\underline{\chi}, t) \underline{\tau}_{2 e q}+\alpha_{2} \underline{e}_{4}(t)+\underline{f_{2}}(\underline{\chi}, \underline{\dot{\chi}}, t)=\underline{0}$.

Now considering the whole crane system, given the condition $\lim _{t \rightarrow+\infty} \underline{s}(t)=0$ of the second-level sliding surface

$\underline{s}(t)=\lambda_{1} \underline{s}_{1}(t)+\lambda_{2} \underline{s}_{2}(t)$, the Lyapunov function candidate can be presented by

$\underline{V}(\underline{s}(t))=\frac{1}{2} \underline{s}^{T}(t) \underline{s}(t)$.

In other words,

$$
\begin{aligned}
& \underline{\dot{V}}(t)=\underline{s}^{T}(t)\left(\lambda_{1} \underline{\dot{s}}_{1}(t)+\lambda_{2} \underline{\dot{s}}_{2}(t)\right) \\
& =\underline{s}^{T}(t)\left\{\begin{array}{l}
\lambda_{1}\left\{\begin{array}{l}
\alpha_{1} \underline{e}_{2}(t)+\underline{f_{1}}(\underline{\chi}, \underline{\dot{\chi}}, t)+ \\
+K_{1}(\underline{\chi}, t) \underline{\tau}-\underline{\ddot{x}}_{1 d}(t)+
\end{array}\right\} \\
+\lambda_{2}\left(\alpha_{2} \underline{e}_{4}(t)+\underline{f_{2}}(\underline{\chi}, \underline{\dot{\chi}}, t)+K_{2}(\underline{\chi}, t) \underline{\tau}\right)
\end{array}\right\} .
\end{aligned}
$$

If the control signal of the second subsystem is also presented by two components of $\underline{\tau}_{1 e q}(t)$ and $\underline{\tau}_{1 s w}(t)$, then

$\underline{\dot{V}}(t)=$

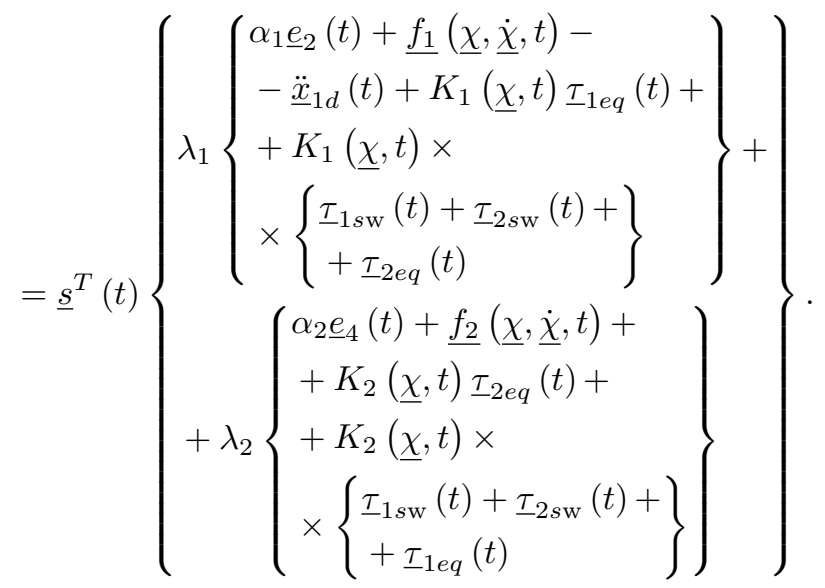

$\Rightarrow \underline{\dot{V}}(t)=\underline{s}^{T}(t)\left\{\begin{array}{l}\lambda_{1} K_{1}(\underline{\chi}, t) \underline{\tau}_{2 e q}(t)+ \\ +\lambda_{2} K_{2}(\underline{\chi}, t) \underline{\tau}_{1 e q}(t)+ \\ +\left(\lambda_{1} K_{1}(\underline{\chi}, t)+\lambda_{2} K_{2}(\underline{\chi}, t)\right) \times \\ \times \underline{\tau}_{1 s \mathrm{w}}(t)+ \\ +\left(\lambda_{1} K_{1}(\underline{\chi}, t)+\lambda_{2} K_{2}(\underline{\chi}, t)\right) \times \\ \times \underline{\tau}_{2 s \mathrm{w}}(t)\end{array}\right\}$.

$\Leftrightarrow \underline{\dot{V}}(t)=\underline{s}^{T}(t)\left\{\begin{array}{l}\lambda_{1} K_{1}(\underline{\chi}, t) \underline{\tau}_{2 e q}(t)+ \\ +\lambda_{2} K_{2}(\underline{\chi}, t) \underline{\tau}_{1 e q}(t)+ \\ +\left(\lambda_{1} K_{1}(\underline{\chi}, t)+\lambda_{2} K_{2}(\underline{\chi}, t)\right) \times \\ \times\left(\underline{\tau}_{1 s \mathrm{w}}(t)+\underline{\tau}_{2 s \mathrm{w}}(t)\right) \\ +\eta_{3} \underline{s}(t)+\eta_{4} \operatorname{sign}(\underline{s}(t))- \\ -\eta_{3} \underline{s}(t)-\eta_{4} \operatorname{sign}(\underline{s}(t))\end{array}\right\}$.

Therefore, the control signal for the 3D overhead crane can be specified by

$\underline{\tau}=\underline{\tau}_{1 e q}(t)+\underline{\tau}_{1 s w}(t)+\underline{\tau}_{2 e q}(t)+\underline{\tau}_{2 s w}(t)$. 
If one chooses

$\left\{\begin{array}{l}\left(\lambda_{1} K_{1}(\underline{\chi}, t)+\lambda_{2} K_{2}(\underline{\chi}, t)\right) \times \\ \times\left(\underline{\tau}_{1 s \mathrm{w}}(t)+\underline{\tau}_{2 s \mathrm{w}}(t)\right) \\ +\lambda_{1} K_{1}(\underline{\chi}, t) \underline{\tau}_{2 e q}(t)+ \\ +\lambda_{2} K_{2}(\underline{\chi}, t) \underline{\tau}_{1 e q}(t)+ \\ +\eta_{3} \underline{s}(t)+\eta_{4} \operatorname{sign}(\underline{s}(t))\end{array}\right\}=\underline{0}$,

which leads to

$\underline{\tau}_{1 s \mathrm{w}}(t)+\underline{\tau}_{2 s \mathrm{w}}(t)=$

$=\left\{\begin{array}{c}-\left(\lambda_{1} K_{1}(\underline{\chi}, t)+\lambda_{2} K_{2}(\underline{\chi}, t)\right)^{-1} \times \\ \times\left\{\begin{array}{c}\lambda_{1} K_{1}(\underline{\chi}, t) \underline{\tau}_{2 e q}(t) \\ +\lambda_{2} K_{2}(\underline{\chi}, t) \underline{\tau}_{1 e q}(t) \\ +\eta_{3} \underline{s}(t)+\eta_{4} \operatorname{sign}(\underline{s}(t))\end{array}\right\}\end{array}\right\}$,

then it has

$\underline{\tau}_{1 s \mathrm{w}}(t)+\underline{\tau}_{2 s \mathrm{w}}(t)=$
$=\left\{\begin{array}{c}-\left(\lambda_{1} K_{1}(\underline{\chi}, t)+\lambda_{2} K_{2}(\underline{\chi}, t)\right)^{-1} \times \\ \times\left\{\begin{array}{c}\lambda_{1} K_{1}(\underline{\chi}, t) \underline{\tau}_{2 e q}(t)+ \\ +\lambda_{2} K_{2}(\underline{\chi}, t) \underline{\tau}_{1 e q}(t)+ \\ +\eta_{3} \underline{s}(t)+\eta_{4} \operatorname{sign}(\underline{s}(t))\end{array}\right\}\end{array}\right\}$.

In other words, substituting (48) into (47) completes the proof.

It is noted that in the proposed control law (28) it may appear the phenomenon of oscillation on the sliding surface due to the change in sign $(\underline{s}(t))$. To mitigate the issue, it is proposed to utilize the function sat $(\underline{s}(t))$ rather than sign $(\underline{s}(t))$. The function sat $(\underline{s}(t))$ is specified by

$\operatorname{sat}(\underline{s}(t))=\left\{\begin{array}{l}\operatorname{sign}(\underline{s}(t)) \text { if }|\underline{s}(t)| \geq 1 \\ \underline{s}(t) \text { if }|\underline{s}(t)|<1 .\end{array}\right.$

\section{Adaptive Fuzzy Learning Scheme}

It can be seen in the analysis of Section 3 that if the parameters $\alpha_{1}, \alpha_{2}, \lambda_{1}$ and $\lambda_{2}$ are fixed, it causes vibration in the control system. In order to address the issue, in this work we propose to adaptively learn these parameters over time by the use of a fuzzy system. To simplify the learning process, it is proposed to learn $\alpha_{1}$ only. The other parameters are adapted through $\alpha_{1}$ accordingly as follows.

$$
\alpha_{2}=A_{2} \cdot \alpha_{1}, \lambda_{1}=A_{3} \cdot \alpha_{1}, \lambda_{2}=A_{4} \cdot \alpha_{1},
$$

where $A_{i}$ is a predefined diagonal positive matrix. It is noted that varying $A_{i}$ leads to change of the sliding surfaces. And if $A_{i}$ is properly chosen, the control system states move on the sliding surfaces to converge to zero,
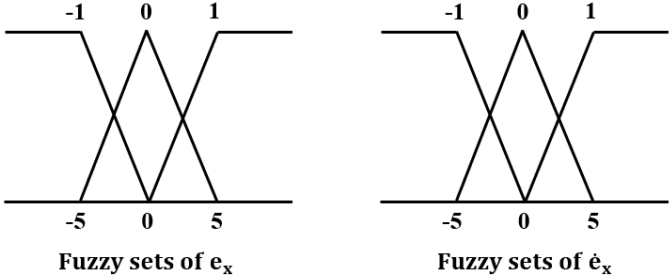

(a) Learning $a$ given $e_{x}$ and $\dot{e_{x}}$
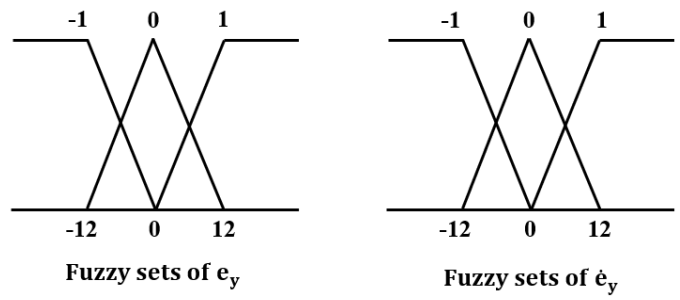

(b) Learning $b$ given $e_{y}$ and $e_{y}$
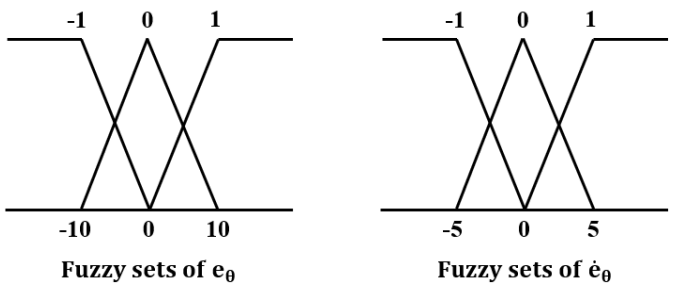

(c) Learning $c$ given $\theta$ and $\dot{\theta}$

Fig. 3: The fuzzy sets of the input language variables.

which effectively minimizes the chattering phenomena in the under-actuated crane systems.

We now investigate how to learn the matrix $\alpha_{1}$. Let us assume $\alpha_{1}$ has the following form.

$$
\alpha_{1}=\left[\begin{array}{lll}
a & 0 & 0 \\
0 & b & 0 \\
0 & 0 & c
\end{array}\right],
$$

where $a, b, c$ are the parameters that will be adaptively learned by using the fuzzy inference rule system.

The parameter $a, b, c$ are learned through a fuzzy logic system given the input language variables $e_{x}=$ $x-x_{d}$ and $\dot{e}_{x}, e_{y}=y-y_{d}$ and $\dot{e}_{y}, e_{\theta}=\theta-\theta_{d}$ and $\dot{e}_{\theta}$, respectively. It is noted that each input language variable consists of a three triangular fuzzy set $\left[\begin{array}{lll}-1 & 0 & 1\end{array}\right]$. The continuous functions of the input language variables are shown in Fig. 3. By using the Sugeno model, the output variables are presented by $\left[\begin{array}{lllll}-2 & -1 & 0 & 1 & 2\end{array}\right]$, which corresponds to

$\left[\begin{array}{lllll}a_{1} & a_{2} & a_{3} & a_{4} & a_{5}\end{array}\right]=\left[\begin{array}{lllll}0.2 & 0.4 & 0.6 & 0.4 & 0.2\end{array}\right]$ for learning $a$,

$\left[\begin{array}{lllll}b_{1} & b_{2} & b_{3} & b_{4} & b_{5}\end{array}\right]=\left[\begin{array}{lllll}0.1 & 0.3 & 0.5 & 0.1 & 0.1\end{array}\right]$ for learning $b$, and $\left[\begin{array}{lllll}c_{1} & c_{2} & c_{3} & c_{4} & c_{5}\end{array}\right]=\left[\begin{array}{lllll}15 & 10 & 5 & 10 & 15\end{array}\right]$ for learning $c$. The fuzzy inference rule system is shown in Table 1.

Therefore, structure of the hierarchical sliding mode control system for the $3 \mathrm{D}$ overhead crane given the 
Table 1: The inference fuzzy system for learning $a, b, c$.

\begin{tabular}{|c|c|c|c|c|}
\hline \multirow{2}{*}{\multicolumn{2}{|c|}{ Parameter $(a, b, c)$}} & \multicolumn{3}{|c|}{$e_{i}$} \\
\hline & & -1 & 0 & 1 \\
\hline \multirow{3}{*}{$\dot{e}_{i}$} & -1 & 5 & 1 & $\overline{0}$ \\
\hline & 0 & 1 & 0 & -1 \\
\hline & 1 & 0 & -1 & 2 \\
\hline
\end{tabular}

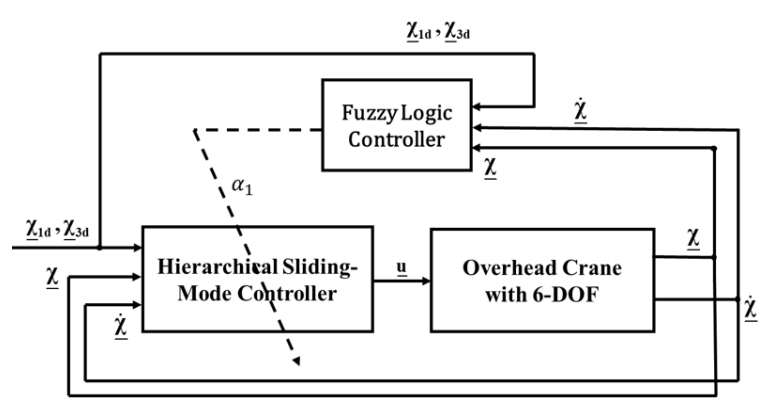

Fig. 4: Control structure of adaptive fuzzy system.

adaptive fuzzy learning scheme is now depicted in Fig. 4.

\section{Results and Discussions}

In order to demonstrate effectiveness of our proposed approach, the adaptive fuzzy hierarchical sliding mode controller (FuzzyHSMC), in controlling the 3D overhead crane with 6 DoF, we conducted the experiments in the synthetic simulation environment. The obtained results are presented in this section. It is noticed that the control performance is measured how effectively the crane can move to a desired location given the proposed control algorithm. In other words, it is expected positions of the trolley, bridge and pulley can reach to the reference values while swing angles of the hoisting cable around a vertical line as well as oscillation of the hoisting cable in the cable direction due to the elasticity are minimized in reasonable time frame.

It is noted that in the experiments, the overhead crane was expected to transport a heavy payload of $5000 \mathrm{~kg}$. The parameters of the crane system are summarized in Table 2. The parameters of the controllers are also encapsulated in Table 3. For the comparison purpose, there were two controllers including HSMC as presented in Section 3 and the proposed FuzzyHSMC implemented in the experiments. It is noticed that the HSMC is a deterministic control scheme where all the parameters of the controller are required to be known. However, in practice the control parameters are unknown and uncertain, which makes the HSMC impractical. In our proposed control approach, the parameters of the FuzzyHSMC are adaptively learned over time
Table 2: The parameters of the 3D overhead crane.

\begin{tabular}{|c|c|c|}
\hline Parameter & Value & Unit \\
\hline$m_{b}$ & 2316.5 & $\mathrm{~kg}$ \\
\hline$m_{c}$ & 5000 & $\mathrm{~kg}$ \\
\hline$m_{t}$ & 371.9 & $\mathrm{~kg}$ \\
\hline $\mathrm{J}$ & 180 & $\mathrm{~kg} \cdot \mathrm{m}^{2}$ \\
\hline $\mathrm{r}$ & 0.31 & $\mathrm{~m}$ \\
\hline $\mathrm{g}$ & 9.81 & $\mathrm{~m} / \mathrm{s}^{2}$ \\
\hline$\rho$ & 300000 & $\mathrm{~N} / \mathrm{m}$ \\
\hline$\Delta \gamma$ & 0.01 & $\mathrm{~m}$ \\
\hline$\eta_{b}$ & 350 & $\mathrm{~N} . \mathrm{m} / \mathrm{s}$ \\
\hline$\eta_{t}$ & 310 & $\mathrm{~N} . \mathrm{m} / \mathrm{s}$ \\
\hline$\eta_{m}$ & 170 & $\mathrm{~N} . \mathrm{m} / \mathrm{s}$ \\
\hline$\eta_{r}$ & 260 & $\mathrm{~N} . \mathrm{m} / \mathrm{s}$ \\
\hline
\end{tabular}

Table 3: The parameters of the controllers.

\begin{tabular}{|c|l|}
\hline Parameter & Value \\
\hline$\lambda_{1}$ & $\operatorname{diag}\left(\left[\begin{array}{lll}0.6 & 0.5 & 4\end{array}\right]\right)$ \\
\hline$\lambda_{2}$ & $\operatorname{diag}\left(\left[\begin{array}{lll}1.2 & 1.5 & 1\end{array}\right]\right)$ \\
\hline$\alpha_{1}$ & $\operatorname{diag}\left(\left[\begin{array}{lll}0.6 & 0.2 & 1.3\end{array}\right]\right)$ \\
\hline$\alpha_{2}$ & $\operatorname{diag}\left(\left[\begin{array}{lll}2.4 & 0.7 & 0.8\end{array}\right]\right)$ \\
\hline$\eta_{3}$ & $\operatorname{diag}\left(\left[\begin{array}{lll}0.2 & 1.6 & 0.1\end{array}\right]\right)$ \\
\hline$\eta_{4}$ & $\operatorname{diag}\left(\left[\begin{array}{lll}4 & 1.6 & 0.6\end{array}\right]\right)$ \\
\hline$A_{2}$ & $\operatorname{diag}\left(\left[\begin{array}{lll}3 & 5 & 0.05\end{array}\right]\right)$ \\
\hline$A_{3}$ & $\operatorname{diag}\left(\left[\begin{array}{lll}1 & 0.5 & 0.1\end{array}\right]\right)$ \\
\hline$A_{4}$ & $\operatorname{diag}\left(\left[\begin{array}{lll}5 & 1.6 & 0.15\end{array}\right]\right)$ \\
\hline
\end{tabular}

by the use of the inference fuzzy system as presented in Section 4. The learning scheme allows the proposed controller to well adapt to nonlinearity and uncertainty of the real-world crane systems.

To demonstrate the proposed approach can efficiently control a 3D overhead crane with 6 DoF in variety of scenarios, we conducted three typical experiments. In the first the reference levels were set to be constant while in the second the desired positions of the trolley, bridge and pulley were changed during the crane's operation. In the third scenario, we considered influence of external disturbances on the control quality of the crane system and how the proposed control law could address the disturbance issues.

\subsection{Constant Input}

In the first scenario, it was expected the overhead crane to transport the $5000 \mathrm{~kg}$ payload from an initial position $\left[\begin{array}{llllll}0 & 0 & 0 & 0 & 0 & 0\end{array}\right]$ to a desired location where the final positions for the trolley, bridge and pulley are $x_{d}=5$ $\mathrm{m}, y_{d}=10 \mathrm{~m}$ and $\theta_{d}=5 \mathrm{rad}$, respectively. The results obtained by two control algorithms, HSMC and FuzzyHSMC, on the control performances of 6 controlled variables including $x(t), y(t), \theta(t), \xi_{x}(t), \xi_{y}(t)$ and $\gamma(t)$ are depicted in Figures 5 and 6 . It can be 


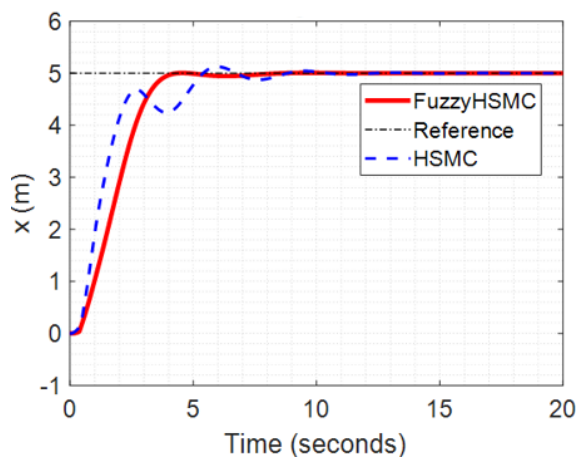

(a) The trolley position $x(t)$

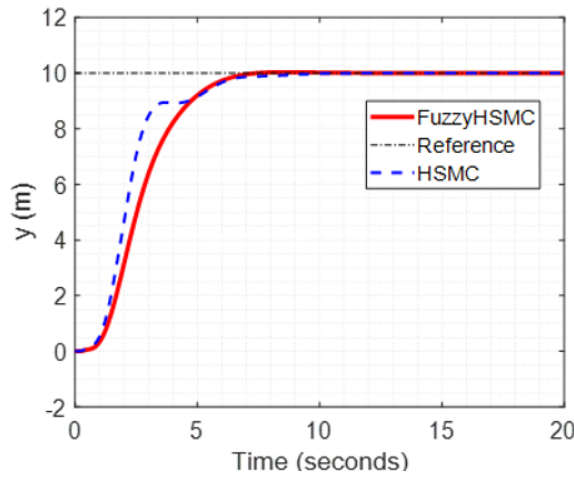

(b) The bridge position $y(t)$

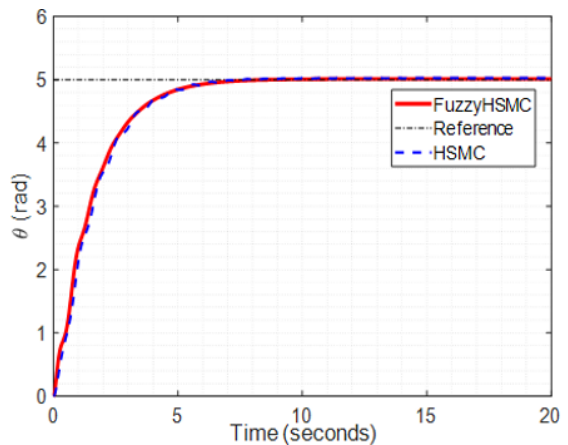

(c) The pulley angle $\theta(t)$

Fig. 5: The actuated states given the constant input.

seen that all the controlled variables could reach $95 \%$ of the reference levels within approximate 6 seconds. More importantly, there is no overshoot in the actuated states while the overshoots in the under-actuated states are relatively small. Under our proposed approach, the overshoots of the sway angles are smaller than 0.1 and 0.3 degrees for $\xi_{x}(t)$ and $\xi_{y}(t)$, respectively, while the oscillation of the hoisting cable is less than $0.2 \mathrm{~cm}$. Overall, while the results obtained by both the controllers in the actuated states are comparable, the proposed control law FuzzyHSMC outperforms the HSMC in the under-actuated components as can be seen in Fig. 6 .

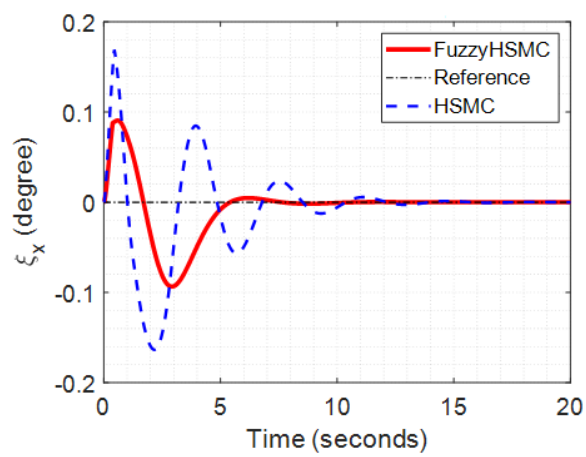

(a) The sway of the payload in $O x z, \xi_{x}(t)$

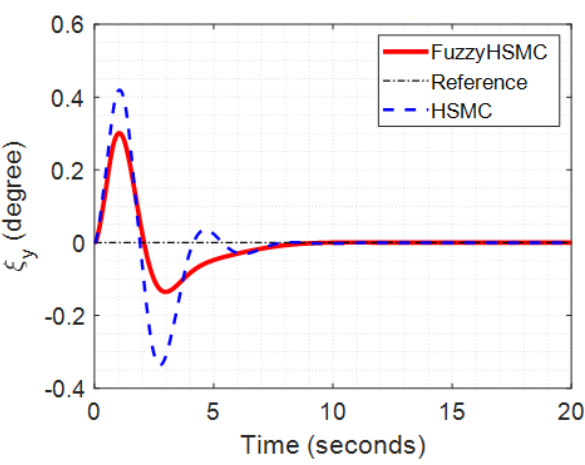

(b) The sway of the payload in $O y z, \xi_{y}(t)$

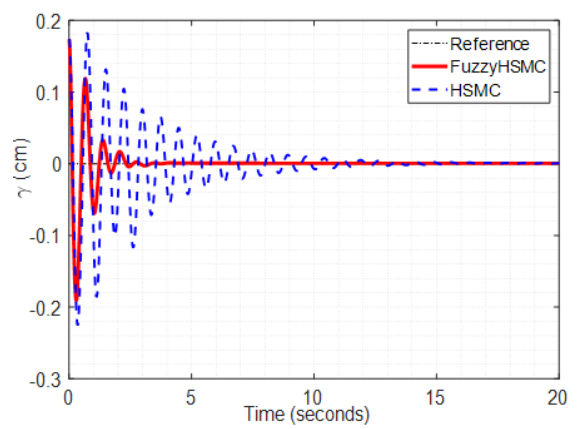

(c) The oscillation of the hoisting cable in the cable direction, $\gamma(t)$

Fig. 6: The under-actuated states given the constant input.

\subsection{Step Input}

In the second scenario, the desired positions of the trolley, bridge and pulley were first set to $x_{d}=2 \mathrm{~m}, y_{d}=6$ $\mathrm{m}$ and $\theta_{d}=2 \mathrm{rad}$, respectively. Nonetheless, at $10 \mathrm{sec}-$ onds from the beginning, the final position of the pulley was then set to $\theta_{d}=6 \mathrm{rad}$. Likewise, at 15 seconds, the final position of the trolley was set to $x_{d}=5 \mathrm{~m}$, and at 20 seconds, the final position of the bridge was set back to $y_{d}=3 \mathrm{~m}$. Though there were changes of the reference levels during the operation of the crane, the control algorithms adapted very well in efficiently controlling motions of the trolley, bridge and pulley 


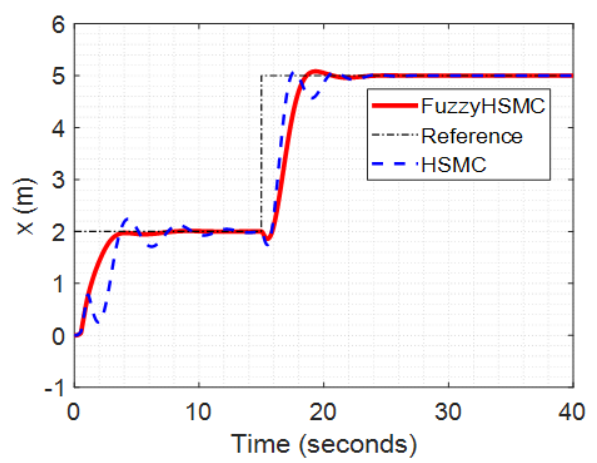

(a) The trolley position $x(t)$

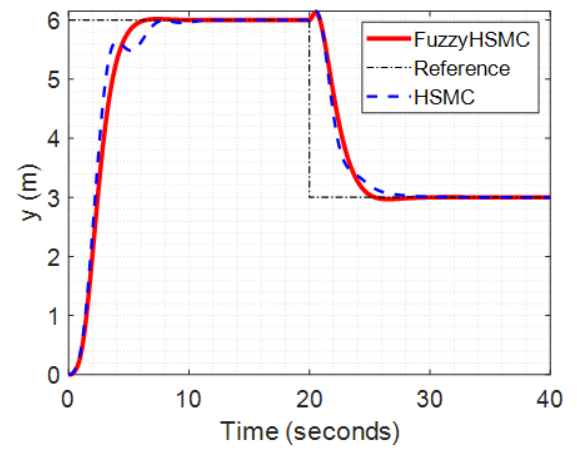

(b) The bridge position $y(t)$

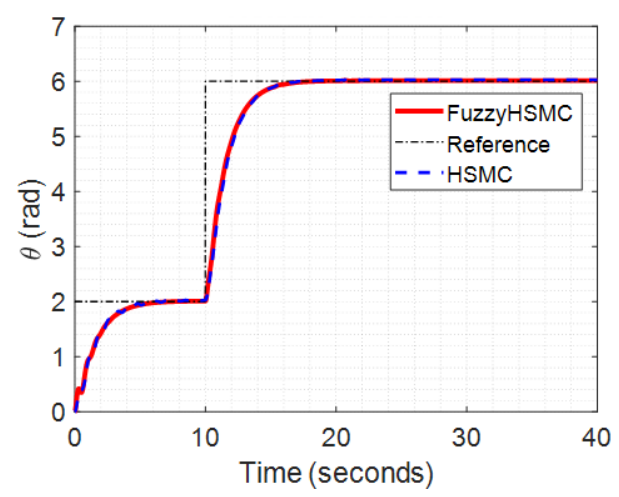

(c) The pulley angle $\theta(t)$

Fig. 7: The actuated states given the step input.

as demonstrated in Fig. 7. Moreover, the changes in the control inputs are also reflected in the responses of the under-actuated components as illustrated in Fig. 8. For instance, though the sway angles and oscillation of the hoisting cable were suppressed almost to zero after about 6 seconds, they re-emerged at the time there were the changes in the control inputs. However, maximum amplitudes of these unexpected swings and oscillation are minor at about 0.1 degrees with respect to $\xi_{x}(t)$ and $\xi_{y}(t)$ and $0.05 \mathrm{~cm}$ with respect to $\gamma(t)$. The underactuated states then went down to zero again after few seconds as can be seen in Fig. 8 .

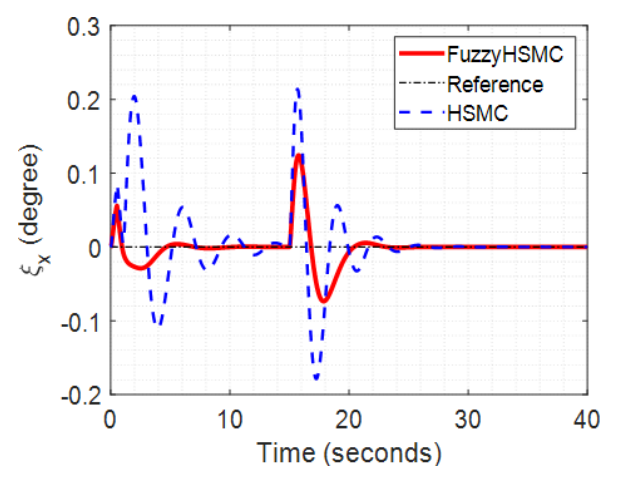

(a) The sway of the payload in $O x z, \xi_{x}(t)$

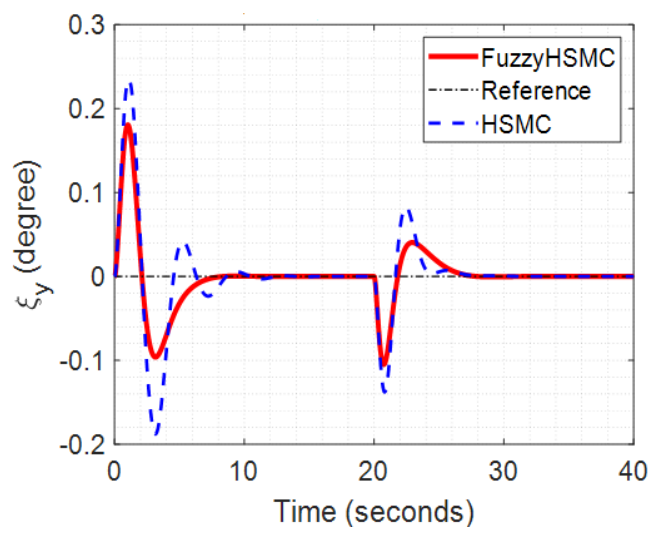

(b) The sway of the payload in $O y z, \xi_{y}(t)$

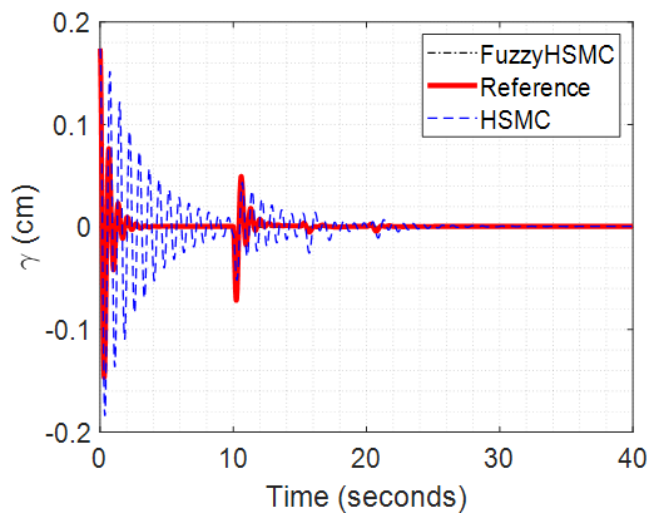

(c) The oscillation of the hoisting cable in the cable direction, $\gamma(t)$

Fig. 8: The under-actuated states given the step input.

\subsection{Noisy Input}

In fact, there always have external disturbances influencing on operations of the real-world crane systems. Hence, in the third experiment, we simulated the external disturbances in the form of pulses injecting into the crane at different time. For instance, at 10 seconds from the beginning, a 2 second width pulse with an amplitude of -500 N.m considered as a noise was injected into the pulley. In a similar fashion, at 15 and 


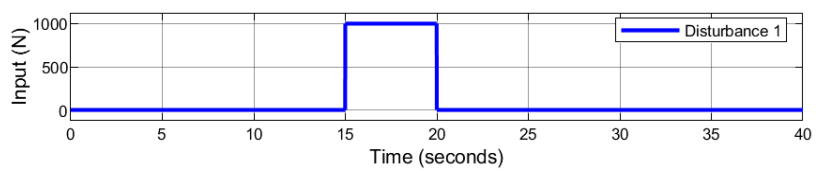

(a) The external disturbance on the trolley

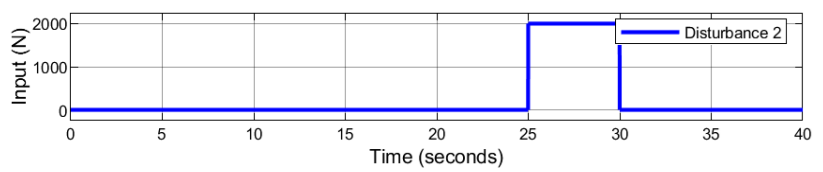

(b) The external disturbance on the bridge

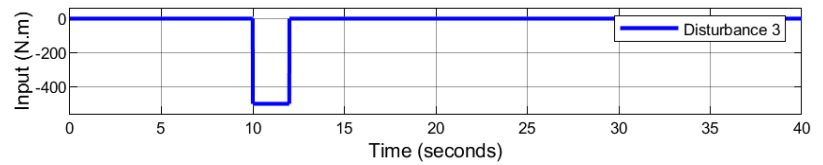

(c) The external disturbance on the pulley

Fig. 9: The external disturbances to the crane system.

25 seconds, two 5 second width pulses with amplitudes of $1000 \mathrm{~N}$ and $2000 \mathrm{~N}$ were injected into the trolley and bridge, respectively. The external disturbances are depicted in Fig. 9.

It is noted that in this third experiment, the final positions of the trolley, bridge and pulley were also set to $x_{d}=5 \mathrm{~m}, y_{d}=10 \mathrm{~m}$ and $\theta_{d}=5 \mathrm{rad}$. The obtained results presenting the control performances are illustrated in Figures 10 and 11. Undoubtedly, it can be seen that the external disturbances caused deterioration of the control performances at the time the disturbances were injected into the crane. However, the controllers quickly steered both the actuated and under-actuated states back to the desired positions. More importantly, as can be seen in Fig. 11, given our control technique, influence caused by the external disturbances on the under-actuated components is insignificant.

Given the results in all three scenarios, it can be seen that since the proposed control algorithm FuzzyHSMC can adaptively learn its parameters over time, it outperforms the deterministic control scheme HSMC.

\section{Conclusions}

A 3D overhead crane with 6 DoF has been considered in this paper, where elasticity of its hoisting cable is taken into account. Oscillation caused by the cable elasticity constitutes the $6^{\text {th }}$ under-actuated output in the crane system. In order to design a controller to effectively control the 6 DoF overhead crane, the HSMC technique is exploited. Moreover, the parameters of the HSMC scheme are proposed to be adaptively learned by utilizing the fuzzy inference rule mechanism, where the

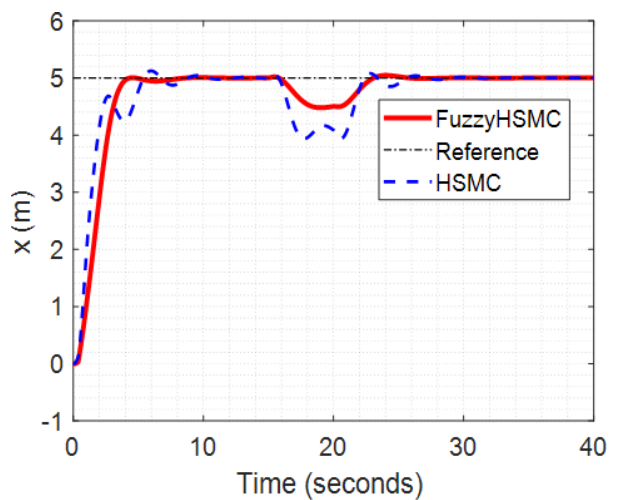

(a) The trolley position $x(t)$

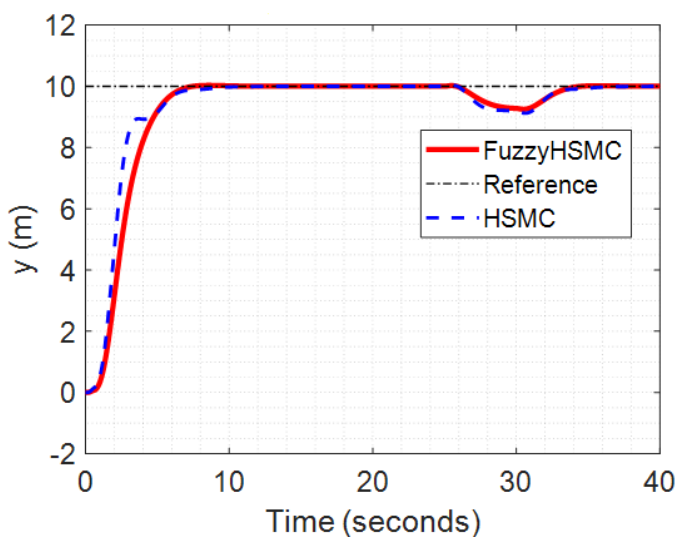

(b) The bridge position $y(t)$

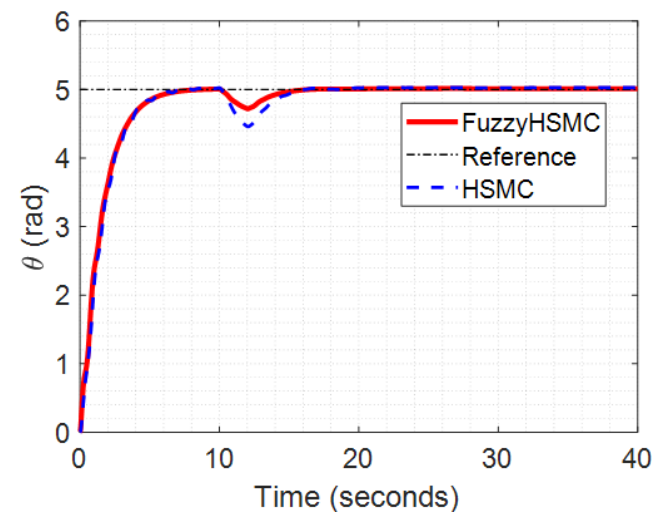

(c) The pulley angle $\theta(t)$

Fig. 10: The actuated states under the external disturbances.

proposed controller is then called FuzzyHSMC. More importantly, stability of the crane controlled by the proposed algorithm is theoretically proved by the use of the Lyapunov function, which guarantees effectiveness of the proposed method. The algorithm was then extensively evaluated, where the obtained results are highly promising. 


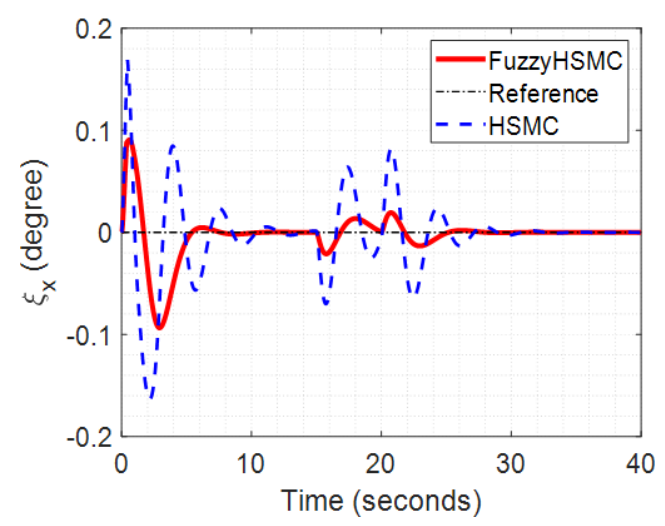

(a) The sway of the payload in $O x z, \xi_{x}(t)$

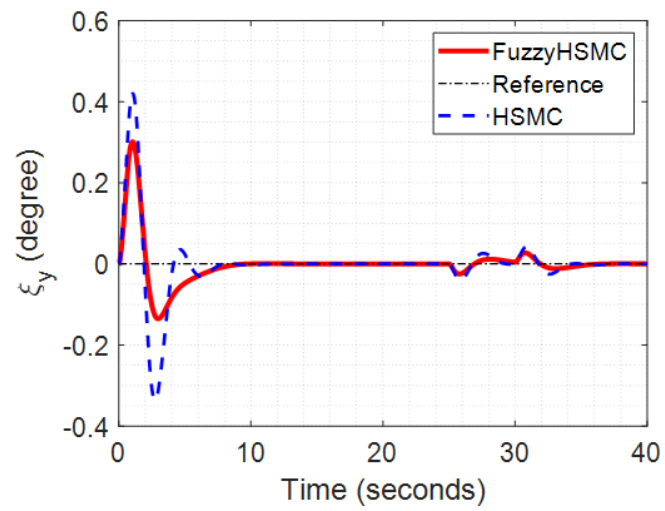

(b) The sway of the payload in $O y z, \xi_{y}(t)$

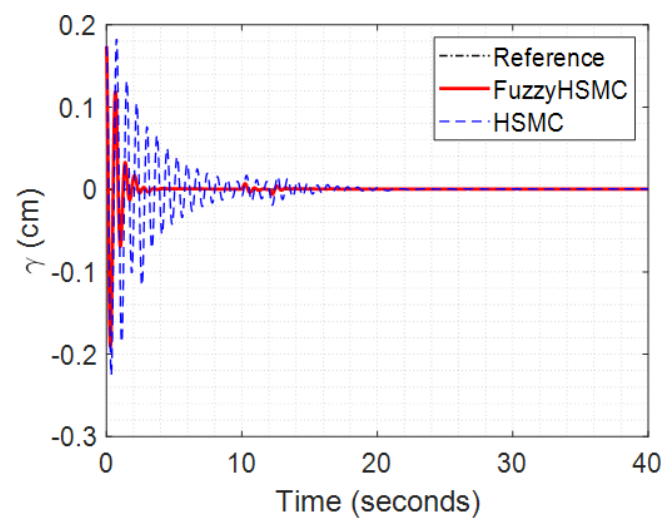

(c) The oscillation of the hoisting cable in the cable direction, $\gamma(t)$

Fig. 11: The under-actuated states under the external disturbances.

\section{Appendix}

The coefficients of the matrix $M(\chi, t)$ are computed as follows. $m_{11}=m_{c}+m_{t}$.

$m_{21}=0$.

$m_{12}=m_{21}$.

$m_{31}=m_{c} r \sin \left(\xi_{x}(t)\right), m_{13}=m_{31}$.

$m_{14}=m_{c}\left(\gamma(t)+\Delta \gamma+r\left(\theta(t)+\xi_{x}(t)\right)\right) \cos \left(\xi_{x}(t)\right)$.

$$
\begin{aligned}
& m_{14}=m_{41} . \\
& m_{51}=m_{15}=0 . \\
& m_{61}=m_{16}=m_{c} \sin \left(\xi_{x}(t)\right) . \\
& m_{22}=m_{c}+m_{b}+m_{t} . \\
& m_{32}=m_{23}=m_{c} r \cos \left(\xi_{x}(t)\right) \sin \left(\xi_{y}(t)\right) .
\end{aligned}
$$$$
m_{42}=m_{24}=\left\{\begin{array}{l}
m_{c} r c o s\left(\xi_{x}(t)\right) \sin \left(\xi_{y}(t)\right)- \\
-m_{c} \gamma(t) \sin \left(\xi_{x}(t)\right) \sin \left(\xi_{y}(t)\right)- \\
-m_{c} r \theta(t) \sin \left(\xi_{x}(t)\right) \sin \left(\xi_{y}(t)\right)- \\
-m_{c} \Delta \gamma \sin \left(\xi_{x}(t)\right) \sin \left(\xi_{y}(t)\right)- \\
-m_{c} r \xi_{x}(t) \sin \left(\xi_{x}(t)\right) \sin \left(\xi_{y}(t)\right)
\end{array}\right\} .
$$

$m_{52}=m_{25}=$$$
=m_{c}\left\{\begin{array}{l}
\gamma(t)+\Delta \gamma+ \\
+r\left(\theta(t)+\xi_{x}(t)\right)
\end{array}\right\} \cos \left(\xi_{x}(t)\right) \cos \left(\xi_{y}(t)\right) .
$$$$
m_{62}=m_{26}=m_{c} \cos \left(\xi_{x}(t)\right) \sin \left(\xi_{y}(t)\right) \text {. }
$$$$
m_{33}=\left\{\begin{array}{l}
J+m_{c} r^{2} \cos ^{2}\left(\xi_{x}(t)\right) \cos ^{2}\left(\xi_{y}(t)\right)+ \\
+m_{c} r^{2} \cos ^{2}\left(\xi_{x}(t)\right) \sin ^{2}\left(\xi_{y}(t)\right)+ \\
+m_{c} r^{2} \sin ^{2}\left(\xi_{x}(t)\right)
\end{array}\right\} .
$$$$
m_{43}=m_{34}=
$$$$
=\left\{\begin{array}{l}
m_{c} \mathrm{r}\left(\gamma(t)+\Delta \gamma+r\left(\theta(t)+\xi_{x}(t)\right)\right) \times \\
\cos \left(\xi_{x}(t)\right) \sin \left(\xi_{x}(t)\right) \\
-m_{c} \mathrm{r}\left(\gamma(t)+\Delta \gamma+r\left(\theta(t)+\xi_{x}(t)\right)\right) \times \\
\cos ^{2}\left(\xi_{x}(t)\right) \sin ^{2}\left(\xi_{x}(t)\right) \\
-m_{c} r^{2} \cos ^{2}\left(\xi_{x}(t)\right) \sin ^{2}\left(\xi_{y}(t)\right)
\end{array}\right\} .
$$$$
m_{53}=m_{35}=\frac{1}{4} m_{c} r^{2} \sin \left(2 \xi_{x}(t)\right) \sin \left(2 \xi_{y}(t)\right) \text {. }
$$$$
m_{63}=m_{36}=m_{c} r .
$$$$
m_{44}=m_{c}\left\{\begin{array}{l}
-r\left(\gamma(t)+\Delta \gamma+r\left(\theta(t)+\xi_{x}(t)\right)\right) \times \\
\sin ^{2}\left(\xi_{y}(t)\right) \sin \left(2 \xi_{x}(t)\right)+ \\
+\left(\gamma(t)+\Delta \gamma+r\left(\theta(t)+\xi_{x}(t)\right)\right)^{2} \times \\
\times \cos ^{2}\left(\xi_{x}(t)\right)+ \\
+\left(\gamma(t)+\Delta \gamma+r\left(\theta(t)+\xi_{x}(t)\right)\right)^{2} \times \\
\times \sin ^{2}\left(\xi_{x}(t)\right) \cos ^{2}\left(\xi_{y}(t)\right)+ \\
+r^{2} \sin ^{2}\left(\xi_{y}(t)\right) \cos ^{2}\left(\xi_{x}(t)\right)+ \\
+\left(\gamma(t)+\Delta \gamma+r\left(\theta(t)+\xi_{x}(t)\right)\right)^{2} \times \\
\times \sin ^{2}\left(\xi_{y}(t)\right) \sin ^{2}\left(\xi_{x}(t)\right)
\end{array}\right\} .
$$$$
m_{54}=m_{c}\left\{\begin{array}{l}
r\left(\gamma(t)+\Delta \gamma+r\left(\theta(t)+\xi_{x}(t)\right)\right) \times \\
\times \sin ^{2}\left(\xi_{y}(t)\right) \sin \left(2 \xi_{x}(t)\right)+ \\
+\left(\gamma(t)+\Delta \gamma+r\left(\theta(t)+\xi_{x}(t)\right)\right)^{2} \times \\
\times \cos ^{2}\left(\xi_{x}(t)\right)+ \\
+\left(\gamma(t)+\Delta \gamma+r\left(\theta(t)+\xi_{x}(t)\right)\right)^{2} \times \\
\times \sin ^{2}\left(\xi_{x}(t)\right) \cos ^{2}\left(\xi_{y}(t)\right)+ \\
+\left(\gamma(t)+\Delta \gamma+r\left(\theta(t)+\xi_{x}(t)\right)\right)^{2} \times \\
\times \sin ^{2}\left(\xi_{y}(t)\right) \sin ^{2}\left(\xi_{x}(t)\right)+ \\
+r^{2} \sin ^{2}\left(\xi_{y}(t)\right) \cos ^{2}\left(\xi_{x}(t)\right)
\end{array}\right\}
$$

$m_{45}=m_{54}$. 


$$
\begin{aligned}
& m_{64}=m_{c} \times \\
& \quad \times\left\{\begin{array}{l}
r \theta(t) \sin ^{2}\left(\xi_{y}(t)\right) \cos \left(\xi_{x}(t)\right) \sin \left(\xi_{x}(t)\right)+ \\
+r \xi_{x}(t) \sin ^{2}\left(\xi_{y}(t)\right) \cos \left(\xi_{x}(t)\right) \sin \left(\xi_{x}(t)\right)+ \\
+\gamma(t) \sin ^{2}\left(\xi_{y}(t)\right) \cos \left(\xi_{x}(t)\right) \sin \left(\xi_{x}(t)\right)+ \\
+\Delta \gamma \sin ^{2}\left(\xi_{y}(t)\right) \cos \left(\xi_{x}(t)\right) \sin \left(\xi_{x}(t)\right)+ \\
+r \sin ^{2}\left(\xi_{y}(t)\right) \cos ^{2}\left(\xi_{x}(t)\right)- \\
-r\left(\theta(t)+\xi_{x}(t)\right) \sin ^{2}\left(\xi_{y}(t)\right) \cos \left(\xi_{x}(t)\right) \times \\
\times \sin \left(\xi_{x}(t)\right)- \\
-\gamma(t) \sin ^{2}\left(\xi_{y}(t)\right) \cos \left(\xi_{x}(t)\right) \sin \left(\xi_{x}(t)\right)- \\
-\Delta \gamma \sin ^{2}\left(\xi_{y}(t)\right) \cos \left(\xi_{x}(t)\right) \sin \left(\xi_{x}(t)\right)
\end{array}\right\} .
\end{aligned}
$$$$
\begin{aligned}
& m_{46}=m_{64} . \\
& m_{55}=m_{c} \times \\
& \quad\left\{\begin{array}{l}
\gamma^{2}(t) \cos ^{2}\left(\xi_{x}(t)\right)+r^{2} \theta^{2}(t) \cos ^{2}\left(\xi_{x}(t)\right)+ \\
+\gamma^{2}(t) \cos ^{2}\left(\xi_{x}(t)\right)+r^{2} \xi_{x}^{2}(t) \cos ^{2}\left(\xi_{x}(t)\right)+ \\
+2 \gamma(t) \Delta \gamma \cos ^{2}\left(\xi_{x}(t)\right)+ \\
+2 r \gamma(t) \theta(t) \cos ^{2}\left(\xi_{x}(t)\right)+ \\
+2 r \gamma(t) \xi_{x}(t) \cos ^{2}\left(\xi_{x}(t)\right)+ \\
+2 \Delta \gamma r \theta(t) \cos ^{2}\left(\xi_{x}(t)\right)+ \\
+2 r^{2} \xi_{x}(t) \theta(t) \cos ^{2}\left(\xi_{x}(t)\right)+ \\
+2 \Delta \gamma r \xi_{x}(t) \cos ^{2}\left(\xi_{x}(t)\right)+ \\
+r^{2} \sin ^{2}\left(\xi_{x}(t)\right) \sin ^{2}\left(\xi_{y}(t)\right)- \\
-r \gamma(t) \sin ^{2}\left(\xi_{y}(t)\right) \sin \left(2 \xi_{x}(t)\right)- \\
-r\left(\Delta \gamma+r \theta(t)+r \xi_{x}(t)\right) \sin ^{2}\left(\xi_{y}(t)\right) \times \\
\times \sin \left(2 \xi_{x}(t)\right)
\end{array}\right\} .
\end{aligned}
$$

$m_{65}=m_{56}=$

$=m_{c} r \cos \left(\xi_{x}(t)\right) \cos \left(\xi_{y}(t)\right) \sin \left(\xi_{x}(t)\right) \sin \left(\xi_{y}(t)\right)$.

$m_{66}=m_{c}$.

Likewise, the coefficients of matrix $C(\chi, \dot{\chi}, t)$ are calculated by $c_{11}=c_{21}=c_{31}=c_{41}=c_{51}=c_{61}=0$. $c_{12}=c_{22}=c_{32}=c_{42}=c_{52}=c_{62}=0$. $c_{13}=2 m_{c} r \dot{\xi}_{x}(t) \cos \left(\xi_{x}(t)\right)$.

$c_{14}=m_{c}\left\{\begin{array}{l}r \dot{\xi}_{x}(t) \cos \left(\xi_{x}(t)\right)- \\ -\gamma(t) \dot{\xi}_{x}(t) \sin \left(\xi_{x}(t)\right)- \\ -\Delta \gamma \dot{\xi}_{x}(t) \sin \left(\xi_{x}(t)\right)- \\ -r \theta(t) \dot{\xi}_{x}(t) \sin \left(\xi_{x}(t)\right)- \\ -r \xi_{x}(t) \dot{\xi}_{x}(t) \sin \left(\xi_{x}(t)\right)\end{array}\right\}$.

$c_{15}=0, c_{16}=2 m_{c} \dot{\xi}_{x}(t) \cos \left(\xi_{x}(t)\right)$.

$c_{23}=2 m_{c} r\left\{\begin{array}{l}\dot{\xi}_{y}(t) \cos \left(\xi_{x}(t)\right) \cos \left(\xi_{y}(t)\right)- \\ -\dot{\xi}_{x}(t) \sin \left(\xi_{x}(t)\right) \sin \left(\xi_{y}(t)\right)\end{array}\right\}$.

$c_{24}=m_{c}\left\{\begin{array}{l}2 r \dot{\xi}_{y}(t) \cos \left(\xi_{x}(t)\right) \cos \left(\xi_{y}(t)\right)- \\ -r \xi_{x}(t) \dot{\xi}_{x}(t) \cos \left(\xi_{x}(t)\right) \sin \left(\xi_{y}(t)\right)- \\ -\gamma(t) \dot{\xi}_{x}(t) \cos \left(\xi_{x}(t)\right) \sin \left(\xi_{y}(t)\right)- \\ -r \theta(t) \dot{\xi}_{x}(t) \cos \left(\xi_{x}(t)\right) \sin \left(\xi_{y}(t)\right)- \\ -\Delta \gamma \dot{\xi}_{x}(t) \cos \left(\xi_{x}(t)\right) \sin \left(\xi_{y}(t)\right)- \\ -2 r \dot{\xi}_{x}(t) \sin \left(\xi_{x}(t)\right) \sin \left(\xi_{y}(t)\right)\end{array}\right\}$.

$$
\begin{aligned}
& c_{25}=-m_{c}\left\{\begin{array}{l}
2 \dot{\xi}_{x}(t) \sin \left(\xi_{x}(t)\right) \cos \left(\xi_{y}(t)\right) \times \\
\times(\gamma(t)+\Delta \gamma)+ \\
+2 r \dot{\xi}_{x}(t) \sin \left(\xi_{x}(t)\right) \cos \left(\xi_{y}(t)\right) \times \\
\times\left(\xi_{x}(t)+\theta(t)\right)+\dot{\xi}_{y}(t) \cos \left(\xi_{x}(t)\right) \times \\
\times \sin \left(\xi_{y}(t)\right)(\gamma(t)+\Delta \gamma)+ \\
+r \dot{\xi}_{y}(t) \cos \left(\xi_{x}(t)\right) \times \\
\times \sin \left(\xi_{y}(t)\right)\left(\left(\xi_{x}(t)+\theta(t)\right)\right)
\end{array}\right\} . \\
& c_{26}=2 m_{c}\left\{\begin{array}{l}
\dot{\xi}_{y}(t) \cos \left(\xi_{x}(t)\right) \cos \left(\xi_{y}(t)\right)- \\
-\dot{\xi}_{x}(t) \sin \left(\xi_{x}(t)\right) \sin \left(\xi_{y}(t)\right)
\end{array}\right\} . \\
& c_{33}=0 .
\end{aligned}
$$$$
c_{34}=m_{c} r\left\{\begin{array}{l}
-\left(\dot{\xi}_{x}(t) \cos ^{2}\left(\xi_{x}(t)\right) \cos ^{2}\left(\xi_{y}(t)\right)\right) \times \\
\times(\gamma(t)+\Delta \gamma)- \\
-r\left(\dot{\xi}_{x}(t) \cos ^{2}\left(\xi_{x}(t)\right) \cos ^{2}\left(\xi_{y}(t)\right)\right) \times \\
\times\left(\xi_{x}(t)+\theta(t)\right)- \\
-r \dot{\xi}_{x}(t) \sin \left(\xi_{x}(t)\right) \cos \left(\xi_{x}(t)\right) \times \\
\times \cos ^{2}\left(\xi_{y}(t)\right)+2 r \cos ^{2}\left(\xi_{x}(t)\right) \times \\
\times \sin \left(\xi_{y}(t)\right) \dot{\xi}_{y}(t) \cos \left(\xi_{y}(t)\right)- \\
-2 r \dot{\xi}_{x}(t) \cos \left(\xi_{x}(t)\right) \sin ^{2}\left(\xi_{y}(t)\right) \times \\
\times \sin \left(\xi_{x}(t)\right)+\dot{\xi}_{x}(t) \cos ^{2}\left(\xi_{x}(t)\right) \times \\
\times \sin ^{2}\left(\xi_{y}(t)\right)(\gamma(t)+\Delta \gamma)+ \\
+r \dot{\xi}_{x}(t) \cos ^{2}\left(\xi_{x}(t)\right) \sin ^{2}\left(\xi_{y}(t)\right) \times \\
\times\left(\xi_{x}(t)+\theta(t)\right)+ \\
+r \dot{\xi}_{x}(t) \cos ^{2}\left(\xi_{x}(t)\right) \sin \left(\xi_{x}(t)\right)- \\
-\dot{\xi}_{x}(t) \sin ^{2}\left(\xi_{x}(t)\right)(\gamma(t)+r \theta(t))- \\
-\dot{\xi}_{x}(t) \sin ^{2}\left(\xi_{x}(t)\right)\left(\Delta \gamma+r \xi_{x}(t)\right)+ \\
+\frac{1}{2} \dot{\xi}_{y}(t) \sin ^{2}\left(2 \xi_{x}(t)\right) \sin \left(2 \xi_{y}(t)\right) \times \\
\times(\gamma(t)+\Delta \gamma)+\frac{1}{2} r \dot{\xi}_{y}(t) \times \\
\times \sin ^{2}\left(2 \xi_{x}(t)\right) \sin \left(2 \xi_{y}(t)\right)\left(\xi_{x}(t)+\theta(t)\right)
\end{array}\right\} .
$$$$
c_{35}=m_{c} r\left\{\begin{array}{l}
r \dot{\xi}_{y}(t) \sin \left(\xi_{x}(t)\right) \cos \left(\xi_{x}(t)\right) \times \\
\times \cos ^{2}\left(\xi_{y}(t)\right)-\dot{\xi}_{y}(t) \cos ^{2}\left(\xi_{x}(t)\right) \times \\
\times \cos ^{2}\left(\xi_{y}(t)\right)(\gamma(t)+\Delta \gamma)- \\
-r \dot{\xi}_{y}(t) \cos ^{2}\left(\xi_{x}(t)\right) \cos ^{2}\left(\xi_{y}(t)\right) \times \\
\times\left(\xi_{x}(t)+\theta(t)-\frac{1}{2} \dot{\xi}_{x}(t) \sin \left(2 \xi_{x}(t)\right) \times\right. \\
\times \sin \left(2 \xi_{y}(t)\right)(\gamma(t)+\Delta \gamma)- \\
-\frac{1}{2} r \dot{\xi}_{x}(t) \sin \left(2 \xi_{x}(t)\right) \sin \left(2 \xi_{y}(t)\right) \times \\
\times\left(\xi_{x}(t)+\theta(t)\right)- \\
-\dot{\xi}_{y}(t) \cos ^{2}\left(\xi_{x}(t)\right) \sin ^{2} \times \\
\times\left(\xi_{y}(t)\right)(\gamma(t)+\Delta \gamma)- \\
-r \dot{\xi}_{y}(t) \cos ^{2}\left(\xi_{x}(t)\right) \sin ^{2}\left(\xi_{y}(t)\right) \xi_{x}(t)- \\
-r \dot{\xi}_{y}(t) \cos ^{2}\left(\xi_{x}(t)\right) \sin ^{2}\left(\xi_{y}(t)\right) \theta(t)
\end{array}\right\} .
$$

$c_{36}=0$

$$
c_{43}=2 m_{c} r\left\{\begin{array}{l}
\dot{\xi}_{x}(t)\left(\gamma(t)+\Delta \gamma+r \xi_{x}(t)+r \theta(t)\right)+ \\
+r \dot{\xi}_{y}(t) \cos ^{2}\left(\xi_{x}(t)\right) \cos \left(\xi_{y}(t)\right) \times \\
\times \sin \left(\xi_{y}(t)\right)-r \dot{\xi}_{x}(t) \cos \left(\xi_{x}(t)\right) \times \\
\times \sin \left(\xi_{x}(t)\right) \sin ^{2}\left(\xi_{y}(t)\right)
\end{array}\right\} .
$$



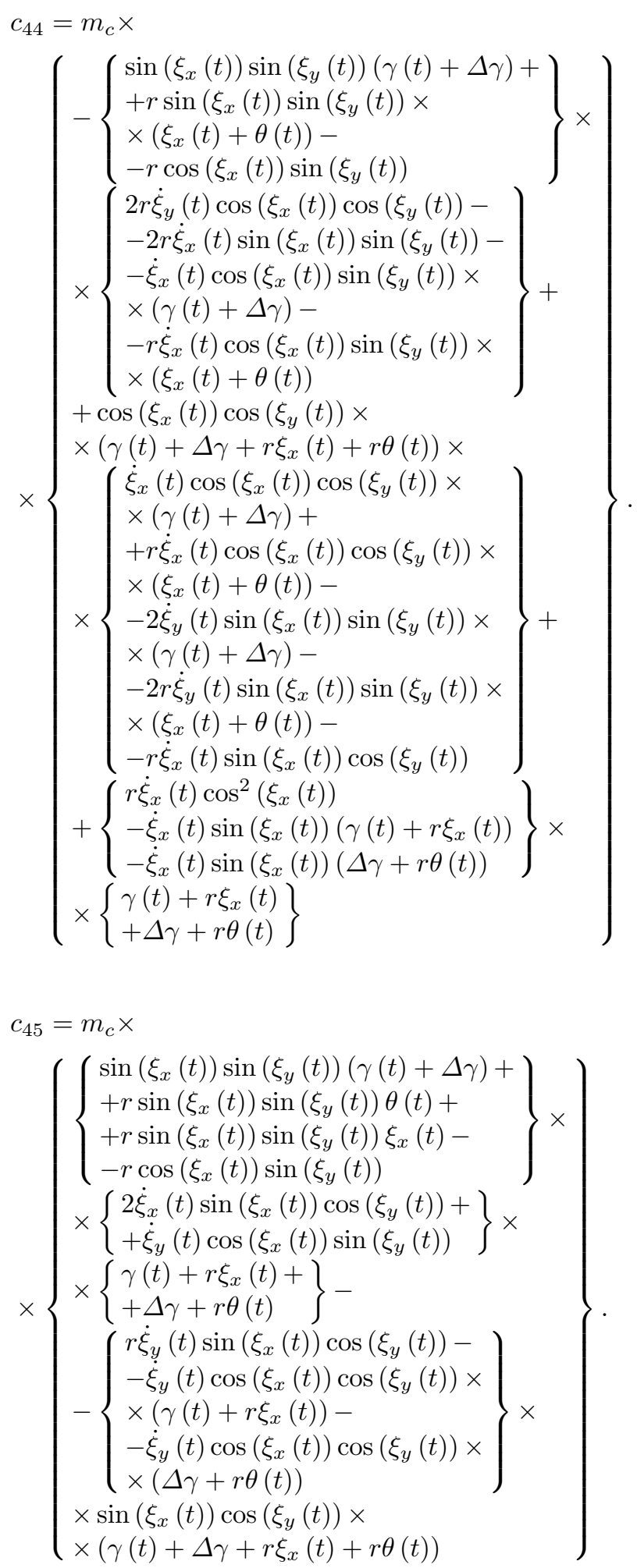

$c_{46}=2 m_{c}\left\{\begin{array}{l}\dot{\xi}_{x}(t)\left(\gamma(t)+\Delta \gamma+r \xi_{x}(t)+r \theta(t)\right)+ \\ +r \dot{\xi}_{y}(t) \cos ^{2}\left(\xi_{x}(t)\right) \cos \left(\xi_{y}(t)\right) \times \\ \times \sin \left(\xi_{y}(t)\right)-r \dot{\xi}_{x}(t) \cos \left(\xi_{x}(t)\right) \times \\ \times \sin \left(\xi_{x}(t)\right) \sin ^{2}\left(\xi_{y}(t)\right)\end{array}\right\}$. $c_{53}=2 m_{c} r\left\{\begin{array}{l}\dot{\xi}_{y}(t) \cos ^{2}\left(\xi_{x}(t)\right) \times \\ \times\left(\gamma(t)+\Delta \gamma+r \xi_{x}(t)+r \theta(t)\right)- \\ -r \dot{\xi}_{x}(t) \sin ^{2}\left(\xi_{x}(t)\right) \cos \left(\xi_{y}(t)\right) \times \\ \times \sin \left(\xi_{y}(t)\right)-r \dot{\xi}_{y}(t) \cos \left(\xi_{x}(t)\right) \times \\ \times \sin \left(\xi_{x}(t)\right) \sin ^{2}\left(\xi_{y}(t)\right)\end{array}\right\}$.

$c_{54}=m_{c} \times$

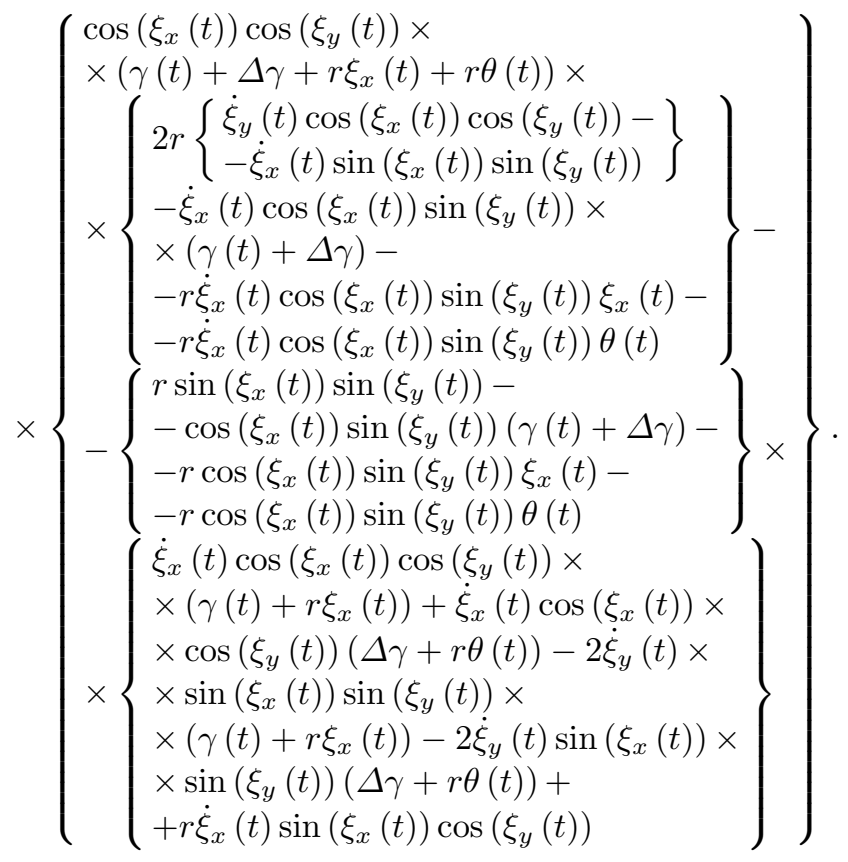

$\left.\begin{array}{l}c_{55}=m_{c} \times \\ \times\left\{\begin{array}{l}r \dot{\xi}_{y}(t) \sin \left(\xi_{x}(t)\right) \cos \left(\xi_{y}(t)\right)- \\ -\dot{\xi}_{y}(t) \cos \left(\xi_{x}(t)\right) \cos \left(\xi_{y}(t)\right) \gamma(t)- \\ -r \dot{\xi}_{y}(t) \cos \left(\xi_{x}(t)\right) \cos \left(\xi_{y}(t)\right) \xi_{x}(t)- \\ -r \dot{\xi}_{y}(t) \cos \left(\xi_{x}(t)\right) \cos \left(\xi_{y}(t)\right) \theta(t)- \\ -\dot{\xi}_{y}(t) \cos \left(\xi_{x}(t)\right) \cos \left(\xi_{y}(t)\right) \Delta \gamma\end{array}\right. \\ \times\left\{\begin{array}{l}r \sin \left(\xi_{x}(t)\right) \sin \left(\xi_{y}(t)\right)- \\ -\cos \left(\xi_{x}(t)\right) \sin \left(\xi_{y}(t)\right) \times \\ \times\left(\gamma(t)+r \xi_{x}(t)\right)- \\ -\cos \left(\xi_{x}(t)\right) \sin \left(\xi_{y}(t)\right) \times \\ \times(\Delta \gamma+r \theta(t))\end{array}\right\}- \\ -\cos \left(\xi_{x}(t)\right) \cos \left(\xi_{y}(t)\right)\left\{\begin{array}{l}\gamma(t)+\Delta \gamma \\ +r \xi_{x}(t)+r \theta(t)\end{array}\right.\end{array}\right\} \times$

$c_{56}=2 m_{c} \times$

$\times\left\{\begin{array}{l}\dot{\xi}_{y}(t) \cos ^{2}\left(\xi_{x}(t)\right) \times \\ \times\left(\gamma(t)+\Delta \gamma+r \xi_{x}(t)+r \theta(t)\right)- \\ -r \dot{\xi}_{x}(t) \sin ^{2}\left(\xi_{x}(t)\right) \cos \left(\xi_{y}(t)\right) \sin \left(\xi_{y}(t)\right)- \\ -r \dot{\xi}_{y}(t) \cos \left(\xi_{x}(t)\right) \sin \left(\xi_{x}(t)\right) \sin ^{2}\left(\xi_{y}(t)\right)\end{array}\right\}$. 


$$
\begin{aligned}
& c_{63}=0, c_{66}=0 . \\
& c_{64}=m_{c} \times \\
& \times\left\{\begin{array}{l}
r \sin \left(\xi_{x}(t)\right) \dot{\xi}_{x}(t) \cos \left(\xi_{x}(t)\right)- \\
-\sin ^{2}\left(\xi_{x}(t)\right) \dot{\xi}_{x}(t)\left(\gamma(t)+r \xi_{x}(t)\right)- \\
-\sin ^{2}\left(\xi_{x}(t)\right) \dot{\xi}_{x}(t)(\Delta \gamma+r \theta(t))- \\
-\left\{\begin{array}{l}
\cos \left(\xi_{x}(t)\right) \cos \left(\xi_{y}(t)\right) \times \\
\times\left(\gamma(t)+r \xi_{x}(t)\right)+ \\
+\cos \left(\xi_{x}(t)\right) \cos \left(\xi_{y}(t)\right) \times \\
\times(\Delta \gamma+r \theta(t))
\end{array}\right\} \times \\
\times\left\{\begin{array}{l}
\dot{\xi}_{x}(t) \cos \left(\xi_{x}(t)\right) \cos \left(\xi_{y}(t)\right)- \\
-2 \dot{\xi}_{y}(t) \sin \left(\xi_{x}(t)\right) \sin \left(\xi_{y}(t)\right)
\end{array}\right\}- \\
-r \dot{\xi}_{x}(t) \cos \left(\xi_{x}(t)\right) \sin \left(\xi_{x}(t)\right) \cos ^{2}\left(\xi_{y}(t)\right)+ \\
+2 r \dot{\xi}_{y}(t) \cos { }^{2}\left(\xi_{x}(t)\right) \cos \left(\xi_{y}(t)\right) \sin ^{2}\left(\xi_{y}(t)\right)- \\
-2 r \dot{\xi}_{x}(t) \cos \left(\xi_{x}(t)\right) \sin \left(\xi_{x}(t)\right) \sin ^{2}\left(\xi_{y}(t)\right)- \\
-\dot{\xi}_{x}(t) \cos ^{2}\left(\xi_{x}(t)\right) \sin ^{2}\left(\xi_{y}(t)\right) \times \\
\times\left(\gamma(t)+r \xi_{x}(t)\right)- \\
-\dot{\xi}_{x}(t) \cos ^{2}\left(\xi_{x}(t)\right) \sin ^{2}\left(\xi_{y}(t)\right)(\Delta \gamma+r \theta(t))
\end{array}\right\} .
\end{aligned}
$$$$
\begin{aligned}
& c_{65}=m_{c} \times \\
& \quad \times\left\{\begin{array}{l}
-\frac{1}{2} \dot{\xi}_{x}(t) \sin \left(2 \xi_{x}(t)\right) \sin \left(2 \xi_{y}(t)\right) \gamma(t)- \\
-\frac{1}{2} r \dot{\xi}_{x}(t) \sin \left(2 \xi_{x}(t)\right) \sin \left(2 \xi_{y}(t)\right) \xi_{x}(t)- \\
-\frac{1}{2} r \dot{\xi}_{x}(t) \sin \left(2 \xi_{x}(t)\right) \sin \left(2 \xi_{y}(t)\right) \theta(t)- \\
-\dot{\xi}_{y}(t) \cos ^{2}\left(\xi_{x}(t)\right) \sin ^{2}\left(\xi_{y}(t)\right) \times \\
\times\left(\gamma(t)+r \xi_{x}(t)\right)- \\
-\dot{\xi}_{y}(t) \cos ^{2}\left(\xi_{x}(t)\right) \sin ^{2}\left(\xi_{y}(t)\right)(\Delta \gamma+r \theta(t))+ \\
+r \dot{\xi}_{y}(t) \cos \left(\xi_{x}(t)\right) \sin \left(\xi_{y}(t)\right) \cos ^{2}\left(\xi_{y}(t)\right)- \\
-\dot{\xi}_{y}(t) \cos ^{2}\left(\xi_{x}(t)\right) \cos ^{2}\left(\xi_{y}(t)\right)(\gamma(t)+\Delta \gamma)- \\
-r \dot{\xi}_{y}(t) \cos ^{2}\left(\xi_{x}(t)\right) \cos ^{2}\left(\xi_{y}(t)\right) \xi_{x}(t)- \\
-r \dot{\xi}_{y}(t) \cos ^{2}\left(\xi_{x}(t)\right) \cos ^{2}\left(\xi_{y}(t)\right) \theta(t)- \\
-\frac{1}{2} \dot{\xi}_{x}(t) \sin \left(2 \xi_{x}(t)\right) \sin \left(2 \xi_{y}(t)\right) \Delta \gamma
\end{array}\right\} .
\end{aligned}
$$

And, the coefficients of vector $G(\chi, t)$ are computed by $g_{1}=m_{c} g r \cos \left(\xi_{x}(t)\right) \cos \left(\xi_{y}(t)\right)$.

$$
\begin{gathered}
g_{2}=-\left\{\begin{array}{l}
m_{c} g \gamma(t) \sin \left(\xi_{x}(t)\right) \cos \left(\xi_{y}(t)\right)+ \\
+m_{c} g \Delta \gamma \sin \left(\xi_{x}(t)\right) \cos \left(\xi_{y}(t)\right)+ \\
+m_{c} g r \theta(t) \sin \left(\xi_{x}(t)\right) \cos \left(\xi_{y}(t)\right)+ \\
+m_{c} g r \xi_{x}(t) \sin \left(\xi_{x}(t)\right) \cos \left(\xi_{y}(t)\right)
\end{array}\right\} . \\
g_{3}=-\left\{\begin{array}{l}
m_{c} g \gamma(t) \sin \left(\xi_{y}(t)\right) \cos \left(\xi_{x}(t)\right)+ \\
+m_{c} g \Delta \gamma \sin \left(\xi_{y}(t)\right) \cos \left(\xi_{x}(t)\right)+ \\
+m_{c} g r \theta(t) \sin \left(\xi_{y}(t)\right) \cos \left(\xi_{x}(t)\right)+ \\
+m_{c} g r \xi_{x}(t) \sin \left(\xi_{y}(t)\right) \cos \left(\xi_{x}(t)\right)- \\
-m_{c} g r \sin \left(\xi_{x}(t)\right) \sin \left(\xi_{y}(t)\right)
\end{array}\right\} .
\end{gathered}
$$

$g_{4}=m_{c} g \cos \left(\xi_{x}(t)\right) \cos \left(\xi_{y}(t)\right)+\rho \Delta \gamma$.

\section{Declarations}

Funding This research was supported by Hanoi University of Industry (14-2021-RD/HD-DHCN).
Conflicts of interest/Competing interests The authors declare that they have no conflict of interest.

Code or data availability Not applicable.

Authors' Contributions Hai Xuan Le, Minh Van Pham and Dong Quoc Hoang developed a model for a 3D overhead crane with 6 degrees of freedom. Dung Manh Do and Hoang Viet Tran designed the controller while Duy Hoang, Hung Van Pham and Linh Nguyen simulated and analysed the results. Minh Van Pham wrote the first draft. Linh Nguyen then edited the final draft. All authors read and approved the final manuscript.

Ethics approval Not applicable.

Consent to participate Not applicable.

Consent for publication Not applicable.

\section{References}

1. T. Van Nguyen, H. X. Le, H. V. Tran, D. A. Nguyen, M. N. Nguyen, and L. Nguyen, "An efficient approach for simo systems using adaptive fuzzy hierarchical sliding mode control," in 2021 IEEE International Conference on Autonomous Robot Systems and Competitions (ICARSC), pp. 85-90, 2021.

2. Y. Li, X. Xi, J. Xie, and C. Liu, "Study and implementation of a cooperative hoisting for two crawler cranes," Journal of Intelligent \& Robotic Systems, vol. 83, p. 165-178, 2016.

3. X. Xing and J. Liu, "Vibration and position control of overhead crane with three-dimensional variable length cable subject to input amplitude and rate constraints," IEEE Transactions on Systems, Man, and Cybernetics: Systems, vol. 51, no. 7, pp. 4127-4138, 2021.

4. A. Campeau-Lecours, S. Foucault, T. Laliberté, B. Mayer-St-Onge, and C. Gosselin, "A cable-suspended intelligent crane assist device for the intuitive manipulation of large payloads," IEEE/ASME Transactions on Mechatronics, vol. 21, no. 4, pp. 2073-2084, 2016.

5. G. Rigatos, P. Siano, and M. Abbaszadeh, "Nonlinear hinfinity control for 4-dof underactuated overhead cranes," Transactions of the Institute of Measurement and Control, vol. 40, no. 7, pp. 2364-2377, 2018.

6. J. Yang and S. Shen, "Novel approach for adaptive tracking control of a 3-d overhead crane system," Journal of Intelligent \& Robotic Systems, vol. 62, pp. 59-80, 2011.

7. G. Boschetti, R. Caracciolo, D. Richiedei, and A. Trevisani, "A non-time based controller for load swing damping and path-tracking in robotic cranes," Journal of Intelligent \& Robotic Systems, vol. 76, p. 201-217, 2014.

8. M. J. Mahmoodabadi and H. K. Haghbayan, "An optimal adaptive hybrid controller for a fourth-order underactuated nonlinear inverted pendulum system," Transactions of the Institute of Measurement and Control, vol. 42 , no. 2 , pp. 285-294, 2020.

9. H. X. Le, L. Nguyen, and K. Thiyagarajan, "A dynamic surface controller based on adaptive neural network for 
dual arm robots," in 2020 15th IEEE Conference on Industrial Electronics and Applications (ICIEA), pp. 555$560,2020$.

10. Q. H. Ngo and K.-S. Hong, "Sliding-mode antisway control of an offshore container crane," IEEE/ASME Transactions on Mechatronics, vol. 17, no. 2, pp. 201-209, 2012.

11. T. V. Nguyen, N. H. Thai, H. T. Pham, T. A. Phan, L. Nguyen, H. X. Le, and H. D. Nguyen, "Adaptive neural network-based backstepping sliding mode control approach for dual-arm robots," Journal of Control, Automation and Electrical Systems, vol. 30, pp. 512-521, Aug 2019.

12. D. Chwa, "Sliding-mode-control-based robust finite-time antisway tracking control of 3-d overhead cranes," IEEE Transactions on Industrial Electronics, vol. 64, no. 8, pp. 6775-6784, 2017.

13. M. I. Al-saedi, "Enhancing the feedforward -feedback controller for nonlinear overhead crane using fuzzy logic controller," IOP Conference Series: Materials Science and Engineering, vol. 745, p. 012074, mar 2020.

14. D. T. Pham, T. V. Nguyen, H. X. Le, L. Nguyen, N. H. Thai, T. A. Phan, H. T. Pham, A. H. Duong, and L. T. Bui, "Adaptive neural network based dynamic surface control for uncertain dual arm robots," International Journal of Dynamics and Control, vol. 8, pp. 824-834, Sep 2020.

15. G. Bartolini, A. Pisano, and E. Usai, "Second-order sliding-mode control of container cranes," Automatica, vol. 38, no. 10, pp. 1783-1790, 2002.

16. L.A.Tuan, J.-J. Kim, S.-G. Lee, T.-G. Lim, and L. C. Nho, "Second-order sliding mode control of a 3D overhead crane with uncertain system parameters," International Journal of Precision Engineering and Manufacturing, vol. 15, no. 5, pp. 811-819, 2014.

17. U. T. T. Hoang, H. X. Le, N. H. Thai, H. V. Pham, and L. Nguyen, "Consistency of control performance in 3D overhead cranes under payload mass uncertainty," Electronics, vol. 9, p. 657, Apr 2020.

18. V.-A. Le, H.-X. Le, L. Nguyen, and M.-X. Phan, "An efficient adaptive hierarchical sliding mode control strategy using neural networks for 3D overhead cranes," International Journal of Automation and Computing, vol. 16, pp. 614-627, Oct 2019.

19. H. X. Le, A. V. Le, and L. Nguyen, "Adaptive fuzzy observer based hierarchical sliding mode control for uncertain 2D overhead cranes," Cyber-Physical Systems, vol. 5, no. 3, pp. 191-208, 2019.

20. X. Weimin, Z. Xiang, L. Yuqiang, Z. Mengjie, and L. Yuyang, "Adaptive dynamic sliding mode control for overhead cranes," in 2015 34th Chinese Control Conference (CCC), pp. 3287-3292, 2015.

21. Y. Li, S. Zhou, and H. Zhu, "A backstepping controller design for underactuated crane system," in 2018 Chinese Control And Decision Conference (CCDC), pp. 28952899, 2018.

22. C.-C. Tsai, H. L. Wu, and K.-H. Chuang, "Backstepping aggregated sliding-mode motion control for automatic 3D overhead cranes," in 2012 IEEE/ASME International Conference on Advanced Intelligent Mechatronics (AIM), pp. 849-854, 2012.

23. W. Wang, J. Yi, D. Zhao, and D. Liu, "Design of a stable sliding-mode controller for a class of second-order underactuated systems," IEE Proceedings - Control Theory and Applications, vol. 151, no. 6, pp. 683-690, 2004.

24. L. A. Zadeh, "Is there a need for fuzzy logic?," Information Sciences, vol. 178, no. 13, pp. $2751-2779,2008$.

25. M. Yue, C. An, Y. Du, and J. Sun, "Indirect adaptive fuzzy control for a nonholonomic/underactuated wheeled inverted pendulum vehicle based on a data-driven trajectory planner," Fuzzy Sets and Systems, vol. 290, pp. 158 - 177, 2016. Theme: Control Engineering and Applications.

26. A. Zdesar, O. Cerman, D. Dovzan, P. Husek, and I. Skrjanc, "Fuzzy control of a helio-crane," Journal of Intelligent \&S Robotic Systems, vol. 72, p. 497-515, 2013.

27. H. Shi, G. Li, X. Bai, and J. Huang, "Research on nonlinear control method of underactuated gantry crane based on machine vision positioning," Symmetry, vol. 11, no. 8, 2019 . 